\title{
GENERALIZED SEMIGROUPS OF QUOTIENTS
}

\author{
BY
}

\author{
C. V. HINKLE, JR.
}

\begin{abstract}
For $S$ a semigroup with 0 and $M_{S}$ a right $S$-set, certain classes of sub $S$-sets called right quotient filters are defined. A study of these right quotient filters is made and examples are given including the classes of intersection large and dense sub $S$-sets respectively. The general semigroup of right quotients $Q$ corresponding to a right quotient filter on a semigroup $S$ is developed and basic properties of this semigroup are noted. A nonzero regular semigroup $S$ is called primitive dependent if each nonzero right ideal of $S$ contains a 0 -minimal right ideal of $S$. The theory developed in the paper enables us to characterize all primitive dependent semigroups having singular congruence the identity in terms of subdirect products of column monomial matrix semigroups over groups.
\end{abstract}

1. Introduction. The classical ring of quotients of a ring $R$ and its generalizations have been topics of major interest in ring theory over the past twenty years with much of the basic ground work laid by Asano [1], Johnson [9], and Utumi [15]. Each type of right quotient ring over $R$ is developed from a given type of filter of right ideals in the ring $R$ and the same general technique is applied in each case.

In this paper generalizations of the se concepts are developed for semigroups and $S$-sets and an attempt is made to unify the theory as much as possible by considering certain classes of sub $S$-sets called right quotient filters. $\$ 3$ is devoted to a study of these right quotient filters. Several examples which are analogues of concepts in ring theory are given including those of intersection large, dense, and strongly dense. We also consider a certain $S$-set extension (semigroup extension) of a semigroup $S$, called a $\mathcal{P}$-quotient set (semigroup) where $\mathcal{P}$ is a right quotient filter on $S$, and we show how $\mathcal{P}$ induces a right quotient filter on such an extension. In certain cases the induced filter is of the same type as that of the original filter.

In $\$ 4$ a generalization of the singular congruence and the torsion relation introduced by Feller and Gantos [6] is given. This generalization, which we call the $P$-torsion congruence on an $S$-set $M$, is defined in terms of an arbitrary right quo-

Received by the editors October 5, 1972.

AMS (MOS) subject classifications (1970). Primary $20 \mathrm{M} 10$.

Key words and phrases. Semigroups of quotients, singular congruences, Rees matrix semigroups, primitive dependent semigroups. 
tient filter $\mathcal{P}$ on $S$, and we are particularly interested in the case where $M$ is $\mathcal{P}$.

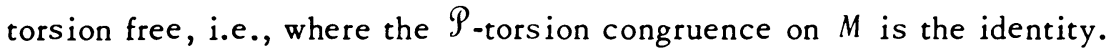

$\$ 5$ is devoted to the study of the general semigroup of right quotients corresponding to a right quotient filter $\mathcal{P}$ on an $S$-set. We begin by giving the basic construction of this semigroup. The main theorems of this section point out the fact

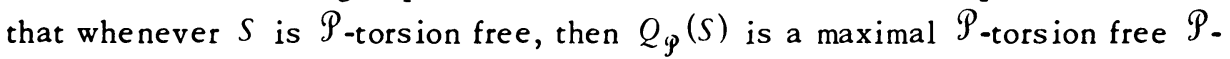
quotient semigroup over $S$ which contains an isomorphic copy over $S$ of every such semigroup extension of $S$. Furthermore, whenever $\mathcal{P}$ is a special filter on $S$ we find that the corresponding quotient semigroup of $Q$ with respect to the induced filter on $Q$ is itself.

As an application of this theory we note that whenever $S$ is $\mathcal{P}_{I}$-torsion free, then $Q_{I}(S)$ is the injective hull of $S$ and is furthermore self-injective. We also restate the well-known theorems concerning the classical semigroup of quotients in terms of the theory developed herein.

In $\$ 6$, we consider semigroups which are 0 -direct unions of semigroups. If a right quotient filter is given on each of the component semigroups of $S$ it is shown that a corresponding right quotient filter is induced on $S$ in a natural way. Here we see that the semigroup of quotients of $S$ is isomorphic to the direct product of the semigroups of quotients on the individual component semigroups. Furthermore, it is shown that every $\mathcal{P}_{\text {-torsion free }} \mathcal{P}_{\text {-quotient semigroup over }} S$ is isomorphic to some subdirect product of $\mathcal{P}_{\text {-torsion free }} \mathcal{P}_{\text {-quotient semigroups over the individ- }}$ ual components.

A nonzero regular semigroup $S$ is called primitive dependent if each nonzero idempotent of $S$ lies above a primitive idempotent of $S$ under the natural partial ordering on the idempotents of $S$. The main purpose of $\$ 7$ is to characterize all primitive dependent semigroups for which the singular congruence is the identity. To this end we first consider completely 0-simple semigroups. A characterization of the singular congruence on a completely 0 -simple semigroup expressed as a regular Rees matrix semigroup is given and it is noted that the semigroup of quotients of such a semigroup is isomorphic to a semigroup of column monomial matrices over a group with 0 . The completely 0 -simple semigroups for which the singular congruence is the identity are then characterized as certain subsemigroups, called $\mathcal{H}$-semigroups, of these semigroups of column monomial matrices. The section is concluded by characterizing the primitive dependent semigroups for which the singular congruence is the identity as certain subdirect products of column monomial matrix semigroups over groups with 0 .

Much of the basic notation and definitions used throughout the paper are given in $\$ 2$. We assume that the reader is familiar with the basic terminology and results on algebraic semigroups employed herein, as presented in Clifford and Preston $[4]$ and $[5]$. 
2. Preliminaries. Throughout this paper each semigroup will contain a zero (0) unless otherwise specified. Let $S$ be a semigroup. A (centered right) $S$-set $M_{S}$ is a set $M$, with an associative scalar operation on $M$ by elements of $S$, which contains an element (necessarily unique) $\theta$ such that $\theta=\theta s=m 0$ for all $m \in M$ and for all $s \in S$. The symbol $\theta$ will be called the zero of $M$. Since the distinction between the zero of $M$ and the zero of $S$ is clear from the context, we shall denote both by the same symbol 0 . Note that if $R$ is a right ideal of $S$ then $R$ becomes an $\bar{S}$-set $R_{S}$ under ordinary multiplication. Also, if $S$ is contained in a semigroup $T$ and if $M_{T}$ is a $T$-set then $M$ becomes an $S$-set $M_{S}$ by restricting the scalar multiplication to the elements of $S$. A sub $S$-set $N_{S}$ of an $S$-set $M_{S}$ is a subset $N$ of $M$ such that $N S \subseteq N$. If $m, n \in M_{S}$ and if $E \subseteq S$ we shall say that $m E$ is pointwise equal to $n E$ when $m s=n s$ for each $s \in E$. This will be denoted as $m E=n E$.

Let $M_{S}$ and $N_{S}$ be $S$-sets. A function $f: M_{S} \rightarrow N_{S}$ is an $S$-homomorphism if, for each $m \in M$ and $s \in S, f(m s)=f(m) s$. The collection of all such $S$-homomorphisms will be denoted by $\operatorname{Hom}_{S}(M, N)$. If there exists $f \in \operatorname{Hom}_{S}(M, N)$ such $f$ is 1-1 and onto then we say $M_{S}$ is $S$-isomorphic to $N_{S}$ and write $M_{S} \approx_{S} N_{S}$. If $M_{S}$ and $N_{S}$ are $S$-sets each containing $A_{S}$ as a sub $S$-set and if there exists an $S$-isomorphism $\phi: M_{S} \rightarrow N_{S}$ such that the restriction $\phi \mid A$ of $\phi$ to $A$ is the identity map on $A$ then we say that " $\phi$ is an $S$-isomorphism over $A_{S}$ " or " $M_{S}$ and $N_{S}$ are $S$-isomorphic over $A_{S}$ ". Corresponding terminology will be used for semigroups and semigroup isomorphisms. All homomorphisms between semigroups will be considered as semigroup homomorphisms unless $S$-homomorphism is clearly indicated. If the semigroups $S$ and $T$ are isomorphic as semigroups we shall write $S \approx T$.

If $f$ is an $S$-homomorphism the domain of $f$ will be denoted by $D_{f}$ and the range of $f$ by $R_{f}$. The zero map from $M_{S}$ will be denoted by 0 and the identitymap on $M$ by $1_{M}$. If $f: M_{S} \rightarrow N_{S}$ and if $A_{S} \subseteq N_{S}$ then $f^{-1}(A)=\{m \in M ; f(m) \in A\}$. The $S$-homomorphism $f$ is called 0 -restricted if $f^{-1}(0)=0$.

An $S$-congruence $\tau$ on $M_{S}$ is an equivalence relation on $M$ such that ( $m s$, $n s) \in \tau$ whenever $(m, n) \in \tau$ for all $s \in S$. The $S$-congruence $\tau$ is said to be 0 -restricted if $(a, 0) \epsilon \tau$ implies $a=0$. If $N_{S}$ is a sub $S_{\text {-set }}$ of $M_{S}$ and if $\rho$ is an $S$. congruence on $M_{S}$ then $\rho \mid N=\rho \cap(N \times N)$ is an $S$-congruence on $N_{S}$.

If $S$ has an identity 1 the $S$-set $M_{S}$ is said to be unital when $m 1=m$ for each $m \in M$. For each semigroup $S$ we shall define $S^{1}$ by $S^{1}=S \cup\{1\}$ where 1 is a symbol not in $S$ and where multiplication on $S$ is extended to $S^{1}$ by defining $1 x=x 1=x$ for each $x \in S^{1}$. With the operation so defined, $S^{1}$ is a semigroup. Note that this definition for $S^{1}$ differs from the standard one. However, with the definition given here each $S$-set $M_{S}$ becomes a unital $S^{1}$-set by defining $m 1=m$ for each $m \in M$. 
The following definitions and theorems are taken from the paper by Berthiaume [3]. A sub $S$-set $N_{S}$ of $M_{S}$ is said to be large (essential) in $M_{S}$ if for each $f \epsilon$ $\operatorname{Hom}\left(M_{S}, K_{S}\right)$ such that $f \mid N$ is $1-1$ then $f$ is $1-1$. In this case $M_{S}$ is called an essential extension of $N_{S}$.

Lemma 2.1. $N_{S}$ is large in $M_{S}$ iff for every $S$-congruence $\rho$ on $M_{S}$ such that $\rho \neq \iota_{M}$ we bave $\rho \mid N \neq \iota_{N}$.

An $S$-set $M_{S}$ is injective if for each $A_{S} \subseteq B_{S}$ and for each $f \in \operatorname{Hom}_{S}(A, M)$ there exists $f^{\prime} \in \operatorname{Hom}_{S}(B, M)$ such that $f^{\prime} \mid A=f$. If $M_{S} \subseteq N_{S}$ and if $N_{S}$ is injective then $N_{S}$ is called an injective extension of $M_{S}$. The following theorem due to Berthiaume guarantees the existence of a minimal injective extension which is unique up to $S$-isomorphism.

Theorem 2.2. The S-set $M_{S}$ is a maximal essential extension of $N_{S}$ iff $M_{S}$ is a minimal injective extension of $N_{S}$. Every $S$-set $N_{S}$ bas such an extension which is unique up to $S$-isomorphism over $N_{S}$.

The minimal injective extension of $N_{S}$ given in the above theorem is called the injective bull of $N_{S}$. Note that $M_{S}$ is the injective hull of $N_{S}$ iff $N_{S}$ is essential in $M_{S}$ and $M_{S}$ is injective.

A semigroup $S$ will be called self-injective if $S_{S}$ is injective.

3. Right quotient filters. The general theory developed throughout this paper is largely dependent on a certain type of filter of sub $S$-sets which will be called a right quotient filter. Several examples of these are given. These examples will later provide applications to the general theory developed herein.

The following definitions are generalizations of corresponding concepts in ring theory. Let $N_{S} \subseteq M_{S}$ where $M_{S}$ is an $S$-set. Then $N_{S}$ is said to be intersection large in $M_{S}$ if for each $0 \neq m \in M$ there exists $s \in S^{1}$ such that $0 \neq m s \in N$. Note that $N_{S}$ is intersection large in $M_{S}$ iff the intersection of $N$ with any nonzero sub $S$-set of $M_{S}$ is always nonzero. A second definition taken from Utumi's paper [15] states that $N_{S}$ is dense in $M_{S}$ iff for each $0 \neq m, n \in M$ there exists $s \in S^{1}$ such that $m s \neq 0$ and $m s \in N$. We easily see that $N_{S}$ is intersection large in $M_{S}$ whenever $N_{S}$ is dense in $M_{S}$. Less generally, $N_{S}$ will be called strongly dense in $M_{S}$ if for each $m_{1}, m_{2}, n \in M$ where $m_{1} \neq m_{2}$ there exists $s \in S^{1}$ such that $m_{1} s \neq m_{2} s$ and $n s \in N$. This latter definition was suggested to the author by J. K. Luedeman for $S$-sets in general. In case $M=S$ and $S$ has an identity this is equivalent to the definition of "dense" as given by F. R. McMorris in [10]. The fourth definition is a generalization of the vital ideal discussed by McMorris in [11]. Let $C$ be any subsemigroup (possibly withour zero) of $S$. Then $N_{S} \subseteq M_{S}$ will be called $C$-vital if for each $0 \neq m \in M$ there exists $c \in C^{1}$ such that 
$0 \neq m c \in N$. Clearly, each $C$-vital sub $S$-set is intersection large in $M_{S}$ and when $C=S$ these two definitions coincide.

The following lemma shows that each of the four concepts given above has the transitive property. In each case the proof follows from the corresponding definition and will be omitted.

Lemma 3.1. Let " $\leq$ " denote any one of the terms "intersection large", "dense", "strongly dense", or "C-vital". Then $X_{S} \leq Y_{S} \leq Z_{S}$ iff $X_{S} \leq Z_{S}$ where $X_{S} \subseteq Y_{S} \subseteq Z_{S}$

Thus " $\leq$ " is a partial order relation on the class of $S$-sets for each definition given above. In the special case where " $\leq$ " represents " $C$-vital", the following theorem shows that " $\leq$ " is preserved under inverse images of $S$-homomorphisms.

Lemma 3.2. Let $M_{S}$ and $N_{S}$ be S-sets and let $\phi \in \operatorname{Hom}_{S}(M, N)$. If $A_{S}$ is $C$ vital in $N_{S}$ then $\phi^{-1}(A)$ is C-vital in $M_{S}$.

Proof. Let $0 \neq m \in M$. If $\phi(m)=0$ then $m 1=m \in \phi^{-1}(A)$. So, suppose $\phi(m) \neq$ 0 . Then there exists $c \in C^{1}$ such that $0 \neq \phi(m) c=\phi(m c) \in A$. Hence $0 \neq m c \epsilon$ $\phi^{-1}(A)$ and the result follows.

Note that if $N_{S}$ is $C$-vital in $M_{S}$ then $m^{-1} N=\{s \in S: m s \in N\}$ is $C$-vital in $S_{s}$ for all $m \in M$. In order to show this, define $\phi_{m}: S \rightarrow M$ by $\phi_{m}(s)=m s$. Then $\phi_{m} \in \operatorname{Hom}_{S}(S, M)$ and $\phi_{m}^{-1}(N)=m^{-1} N$ is $C$-vital in $S_{S}$ by the lemma.

If we restrict ourselves to the class of sub $S$-sets of $M_{S}$ a similar result is valid for the remaining definitions given.

Lemma 3.3. Let " $\leq$ " denote any of the terms "intersection large", "dense", "strongly dense", or "C-vital". Let $A_{S} \leq M_{S}$ and $B_{S} \leq M_{S}$ and let $\phi \in \operatorname{Hom}_{S}(A, M)$. Then $\phi^{-1}(B) \leq M_{S}$.

Proof. By Lemma 3.2 we need only consider "dense" and "strongly dense". Let " $\leq$ " denote "dense" and let $0 \neq m, n \in M$. Then there exists $s_{1} \in S^{1}$ such that $0 \neq m s_{1}$ and $n s_{1} \in A$ and there exists $s_{2} \in S^{1}$ such that $0 \neq m s_{1} s_{2}$ and $f\left(n s_{1}\right) s_{2} \in B$. Therefore, $0 \neq m\left(s_{1} s_{2}\right)$ and $n\left(s_{1} s_{2}\right) \in f^{-1}(B)$ and it follows that $f^{-1}(B) \leq M_{S}$ in this case. The proof is similar when " $\leq$ " denotes "strongly dense".

Let $\mathscr{P}_{I}=\mathscr{P}_{I}\left(M_{S}\right), \mathscr{P}_{D}=\mathscr{P}_{D}\left(M_{S}\right), \mathcal{P}_{S D}=\mathscr{P}_{S D}\left(M_{S}\right)$, and $\mathscr{P}_{C V}=\mathscr{P}_{C V}\left(M_{S}\right)$ denote the classes of intersection large, dense, strongly dense, and $C$-vital sub $S$-sets of $M_{S}$ respectively. In addition, $\mathcal{P}_{B}=\mathscr{P}_{B}\left(M_{S}\right)$ will denote the singleton class $\left\{M_{S}\right\}$. In case $M$ is the semigroup $S$, the subscript " $S$ " will generally be omitted in the notation above. 
In general, a nonempty class of sub $S$-sets of $M_{S}$ is called a right quotient filter (R.Q.F.) of $M_{S}$ if

(1) $A_{S} \in \mathcal{P}$ and $A_{S} \subseteq B_{S} \subseteq M_{S}$ imply $B_{S} \in \mathcal{P}$, and

(2) $A_{S}, B_{S} \in \mathcal{P}$ and $f \in \operatorname{Hom}_{S}(A, M)$ imply $f^{-1}(B) \in \mathcal{P}$.

It is easily seen from Lemma 3.1 and Lemma 3.3 that each of the classes $\mathscr{P}_{I}, \mathscr{P}_{D}, \mathscr{P}_{S D}, \mathscr{P}_{C V}$, and $\mathcal{P}_{B}$ is a right quotient filter on $M_{S}$. These specific filters will be called standard filters.

Lemma 3.4. A right quotient filter $\mathcal{P}$ of $M_{S}$ is closed under finite intersections.

Proof. Let $A_{S}, B_{S} \in \mathcal{P}$ and let $\iota: A \rightarrow M$ be the identity map. Then $A \cap B=$ $\iota^{-1}(B) \in \mathcal{P}$ by property (2) above.

Let $\mathcal{P}$ be a right quotient filter on $M_{S}$. A nonempty subclass $\mathcal{P}^{\prime}$ of $\mathcal{P}$ is said to be a base for $\mathcal{P}$ if for each $A \in \mathcal{P}$ there exists $B \in \mathcal{P}^{\prime}$ such that $B \subseteq A$. Note that a given class of sub $S$-sets of $M_{S}$ can be a base for at most one right quotient filter on $M_{S}$. An arbitrary class $\mathfrak{P}^{\prime}$ of sub $S$-sets will be called a right quotient filter base if it is a base for some right quotient filter $\mathcal{P}$ on $M_{S}$. In this case it is easily seen that $\mathcal{P}^{\prime}$ is a base for the right quotient filter $\mathcal{P}=\left\{A_{S}: A^{\prime} \subseteq A \subseteq\right.$ $M$ for some $A^{\prime} \in \mathcal{P}^{\prime}$ ?. This fact yields the following proposition.

Proposition 3.5. A nonempty class $\mathfrak{P}^{\prime}$ of sub $S$-sets of $M_{S}$ is a right quotient filter base if and only if for eacb $A^{\prime}, B^{\prime} \in \mathcal{P}^{\prime}$ and $f \in \operatorname{Hom}_{S}\left(A^{\prime}, M\right)$ there exists $C^{\prime}$ in $\mathcal{P}^{\prime}$ with $C^{\prime} \subseteq f^{-1}\left(B^{\prime}\right)$.

Note that if $\mathcal{P}$ is a right quotient filter on $M_{S}$ and if $N_{S} \in \mathcal{P}, \mathcal{P} \mid N=\{N \cap$ $A: A \in \mathcal{P}\}$ is a right quotient filter for $N_{S}$ and is also a base for $\mathcal{P}$. In fact, if $\mathcal{P}$ denotes one of the standard filters on $M_{S}$ then $\mathscr{P} \mid N$ is the corresponding standard filter on $N_{S}$.

Let $S$ be a semigroup and let $\mathcal{P}$ be a right quotient filter on $S_{S}$. An $S$-set $M_{S}$

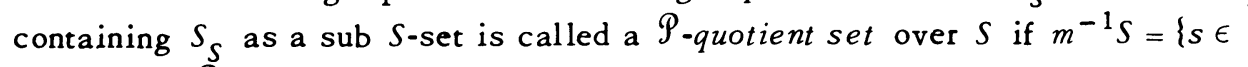
$S: m s \in S\} \in \mathscr{P}$ for each $m \in M$. In case $M$ is a semigroup containing $S$ as a subsemigroup, $M$ is called a $\mathcal{P}$-quotient semigroup over $S$. Clearly, $S$ is always a $\mathcal{P}$. quotient semigroup over itself. Examples of other $\mathscr{P}$-quotient sets are given in the easily proved proposition which follows.

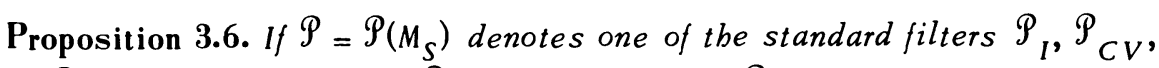
$\mathscr{P}_{D}$, or $\mathscr{P}_{S D}$ on $M_{S}$ and if $S_{S} \in \mathscr{P}\left(M_{S}\right)$ then $M_{S}$ is a $\mathcal{P}$-quotient set over $S$.

In the remainder of this section we shall give sufficient conditions for extending a right quotient filter $\mathcal{P}$ on $S$ to a right quotient filter on any $\mathcal{P}$-quotient semigroup over $S$. This is given below in the special cases where $\mathscr{P}=\mathscr{P}_{I}, \mathscr{P}_{D}$, or $\mathscr{P}_{S D^{\circ}}$ 
Theorem 3.7. Let $S \subseteq T$ where $T$ is a semigroup.

(a) If $S_{S} \in \mathcal{P}_{D}\left(T_{S}\right)$ then $\mathscr{P}^{\prime}=\left\{E T^{1}: E \in \mathscr{P}_{I}(S)\right\}$ is a base for $\mathscr{P}_{I}(T)$.

(b) If $S_{S} \in \mathscr{P}_{D}\left(T_{S}\right)$ then $\mathscr{P}^{\prime}=\left\{E T^{1}: E \in \mathcal{P}_{D}(S)\right\}$ is a base for $\mathcal{P}_{D}(T)$.

(c) If $S_{S} \in \mathcal{P}_{S D}\left(T_{S}\right)$ then $\mathscr{P}^{\prime}=\left\{E T^{1}: E \in \mathscr{P}_{S D}(S)\right\}$ is a base for $\mathcal{P}_{S D}(T)$.

Proof. We shall give the proof of (a). The same general techniques are applied to prove (b) and (c). Let $E \in \mathcal{P}_{I}(S)$ and let $0 \neq t \in T$. Since $E_{S} \in \mathcal{P}_{I}\left(T_{S}\right)$ by Lemma 3.1, there exists $s \in S^{1} \subseteq T^{1}$ such that $0 \neq t s \in E \subseteq E T^{1}$. Thus $E T^{1} \in \mathscr{P}_{\mathrm{I}}(T)$ and it follows that $\mathcal{P}^{\prime} \subseteq \mathcal{P}_{I}(T)$. Now let $E_{T} \in \mathcal{P}_{I}(T)$ and let $E^{\prime}=E \cap S$. We claim that $E^{\prime} \in \mathscr{P}_{I}(S)$. Let $0 \neq s \in S$. Since $E \in \mathcal{P}_{I}(T)$, there exists $t \in T^{1}$ such that $0 \neq s t \epsilon$ $E$. The claim is evident if $t=1$. Otherwise, since $S_{S} \in \mathcal{P}_{D}\left(T_{S}\right)$, there exists $s^{\prime} \epsilon$ $s^{1}$ such that $0 \neq s t s^{\prime}$ and $t s^{\prime} \in S$. Hence we have $0 \neq s\left(t s^{\prime}\right) \in E \cap S=E^{\prime}$ where $\left(t s^{\prime}\right) \in S^{1}$. Thus, $E^{\prime} \in \mathcal{P}_{I}(S)$ and it follows that $E^{\prime} T^{1}=(E \cap S) T^{1} \subseteq E T^{1} \subseteq E$. Therefore, $\mathcal{P}^{\prime}$ is a base for $\mathcal{P}_{I}(T)$.

We can generalize Theorem 3.7 as follows.

Theorem 3.8. Let $S \subseteq T$ where $S$ and $T$ are semigroups. Let $\mathcal{P}^{\prime}\left(T_{S}\right)$ be a base for a right quotient filter $\mathcal{P}\left(T_{S}\right)$ on $T_{S}$. Then $\mathcal{P}^{\prime}(T)=\left\{E T^{1}: E \in \mathcal{P}^{\prime}\left(T_{S}\right)\right\}$ is a base for a right quotient filter $\mathscr{P}(T)$ on $T_{T}$. Furthermore, $\mathcal{P}(T)$ is independent of the base $\mathcal{P}^{\prime}\left(T_{S}\right)$ taken for $\mathcal{P}\left(T_{S}\right)$.

Proof. Let $E_{1} T^{1}$ and $E_{2} T^{1} \in \mathcal{P}^{\prime}(T)$ where $E_{1}$ and $E_{2} \in \mathcal{P}^{\prime}\left(T_{S}\right)$ and let $f \epsilon$ $\operatorname{Hom}_{T}\left(E_{1} T^{1}, T\right)$. Let $f^{\prime}=f \mid E_{1}$. Then $f^{\prime} \in \operatorname{Hom}_{S}\left(E_{1}, T\right)$ and since $\mathcal{P}^{\prime}\left(T_{S}\right)$ is a base for $\mathscr{P}\left(T_{S}\right)$, there exists $E_{3} \in \mathfrak{P}^{\prime}\left(T_{S}\right)$ such that $E_{3} \subseteq f^{\prime-1}\left(E_{2}\right)$. It follows that $E_{3} T^{1} \subseteq f^{\prime-1}\left(E_{2}\right) T^{1} \subseteq f^{-1}\left(E_{2}\right) T^{1} \subseteq f^{-1}\left(E_{2} T^{1}\right)$. Hence, by Proposition 3.5, $\mathcal{P}^{\prime}(T)$ is a filter base on $T_{T}$. Now, let $\overline{\mathcal{P}}_{1}^{\prime}\left(T_{S}\right)$ and $\mathcal{P}_{2}^{\prime}\left(T_{S}\right)$ be any two bases for $\mathcal{P}\left(T_{S}\right)$ and let $\mathscr{P}_{i}^{\prime}(T)=\left\{E T^{1}: E \in \mathcal{P}_{i}^{\prime}\left(T_{S}\right)\right\}, i=1,2$. Let $\mathscr{P}_{1}(T)$ and $\mathcal{P}_{2}(T)$ be the right quotient filters on $T_{T}$ generated by $\mathscr{P}_{1}^{\prime}(T)$ and $\mathscr{P}_{2}^{\prime}(T)$ respectively. If $F \in$ $\mathcal{P}_{1}(T)$ then there exists $E \in \mathcal{P}_{1}^{\prime}\left(T_{S}\right)$ such that $E T^{1} \subseteq F$. Since $\mathcal{P}_{2}^{\prime}\left(T_{S}\right)$ is also a base for $\mathscr{P}\left(T_{S}\right)$, there exists $E^{\prime} \in \mathcal{P}_{2}^{\prime}\left(T_{S}\right)$ such that $E^{\prime} \subseteq E$. Hence $E^{\prime} T^{1} \epsilon$ $\mathcal{P}_{2}(T)$ and $E^{\prime} T^{1} \subseteq E T^{1} \subseteq F$. Therefore, $F \in \mathcal{P}_{2}(T)$ and it follows that $\mathcal{P}_{1}(T) \subseteq$ $\mathscr{P}_{2}(T)$. A symmetrical argument gives the reverse inclusion.

A right quotient filter $\mathscr{P}$ on $S_{S}$ is said to be special if it has the following property:

"If $F$ is a right ideal of $S, E \in \mathcal{P}$, and $x^{-1} F=\{s: x s \in F\} \in \mathcal{P}$ for all $x \in E$, then $F \in \mathcal{P}^{\text {." }}$

Lemma 3.9. Let $\mathcal{P}$ be a right quotient filter on $S_{S}$. Then $\mathcal{P}$ is special iff $\mathcal{P}$ bas the following property:

"For each $E \in \mathcal{P}$ and for each $x \in E$, let $E_{x}$ denote a member of $\mathcal{P}$. Then $E^{\prime}=\bigcup_{x \in E^{x E}} \in \mathcal{P}^{\prime \prime}$ 
Proof. ( $\Rightarrow$ ) If $x \in E$ then $x^{-1} E^{\prime}=\left\{s: x s \in E^{\prime}\right\}$ which contains $E_{x}$ and $E_{x} \epsilon$ $\mathcal{P}$. Hence $x^{-1} E^{\prime} \in \mathcal{P}$ for each $x \in E$ and it follows that $E^{\prime} \in \mathcal{P}$.

$(\Leftarrow)$ Let $E^{\prime}=\bigcup_{x \in E} x\left(x^{-1} F\right)$. Then $E^{\prime} \in \mathcal{P}$ by the hypothesis. However, $E^{\prime} \subseteq F$. Therefore, $F \in \stackrel{\mathcal{P}}{\text { and we have that }} \mathcal{P}$ is special.

Before giving some examples of special quotient filters, we shall digress briefly and consider a semigroup $S$ containing right cancellable elements. Let $C$ be a subsemigroup of the right cancellable elements of $S$. The semigroup $S$ has the right common multiple property (R.C.M.P.) with respect to $C$ if for each $s \epsilon$ $S$ and for each $c \in C, c S \cap s C \neq \square$. Let $\mathscr{P}_{C}=\{R: R$ is a right ideal of $S$ and $R \cap$ $C \neq \square\}$.

Proposition 3.10. $\mathscr{P}_{C}$ is a right quotient filter on $S_{S}$ iff $S$ bas the R.C.M.P. with respect to $C$. In this case, $\mathscr{P}_{C}=\mathscr{P}_{C V}(S)$.

Proof. $(\Rightarrow)$ Let $c \in C$ and $s \in S$. Define $\phi_{s}: S \rightarrow S$ by $\phi_{s}(t)=s t$ for each $t \in S$. Then $\phi_{s} \in \operatorname{Hom}_{S}(S, S)$. Since $c^{2} \in c S \cap C$, we have $c S \in \mathcal{P}_{C}^{S}$. Hence by the definition of an R.Q.F. it follows that $\phi_{s}^{-1}(c S)=\{t \in S: s t \in c S\} \in \mathcal{P}_{C}$. Let $c^{\prime} \epsilon$ $\phi_{s}^{-1}(c S) \cap C$. Then $s c^{\prime} \in c S$ and we have $s C \cap c S \neq \square$. Therefore, $S$ has the R.C.M.P. with respect to $C$.

$(\leftarrow$ ) Now, suppose $S$ has the R.C.M.P. with respect to $C$. We claim that $\mathscr{P}_{C}=\mathscr{P}_{C V}(S)$. Let $R \in \mathscr{P}_{C}$ and let $0 \neq s \in S$. Then there exists $n \in R \cap C$ and there exists $c^{\prime} \in C$ and $s^{\prime} \in S$ such that $s c^{\prime}=n s^{\prime}$. Since $c^{\prime} \in C, s c^{\prime} \neq 0$ and it follows that $s C^{1} \cap R \neq 0$. Therefore, $R \in \mathscr{P}_{C V}(S)$. Now, let $R \in \mathscr{P}_{C V}(S)$ and let $c \in C$. Since $R \in \mathscr{P}_{C V}(S)$, there exists $c^{\prime} \in C^{1}$ such that $c c^{\prime} \in R$. However, $c c^{\prime} \in C$ and we have $R \cap C \neq \square$. Therefore, $R \in \mathcal{P}_{C}$ and the theorem follows.

Several examples of special right quotient filters can now be given as shown in the proposition below.

Proposition 3.11. Let $S$ be a semigroup with zero.

(a) If $S$ contains right cancellable elements and if $S$ bas the R.C.M.P. with respect to a subsemigroup $C$ of the right cancellable elements of $S$ then ${ }^{P}{ }_{C V}(S)$ is a special R.Q.F.

(b) $\mathcal{P}_{B}(S)$ is special iff $S^{2}=S$.

(c) If $\{s \in S \mid s S=0\}=\{0\}$ then $\mathscr{P}_{D}(S)$ is special.

(d) If $\{s \in S \mid s S=0\} \neq\{0\}$ then $\mathscr{P}_{D}(S)=\mathscr{P}_{B}(S)$.

(e) If $S$ is right reductive (i.e. $s S=t S$ implies $s=t$ for $s, t \in S$ ) then

$\mathcal{P}_{S D}(S)$ is special.

(f) If $S$ is not right reductive then $\mathscr{P}_{S D}(S)=\mathscr{P}_{B}(S)$.

Proof. (a) Let $E \in \mathcal{P}_{C V}(S)$ and let $E_{x} \in \mathcal{P}_{C V}(S)$ for each $x \in E$. Since $E \epsilon$ $\mathscr{P}_{C V}$ and since $\mathscr{P}_{C V}=\mathscr{P}_{C}$ by Proposition 3.10, there exists $c \in C \cap E$ and since 
$E_{c} \in P_{C}$, there exists $c^{\prime} \in C \cap E_{c}$. Thus we have $c c^{\prime} \in c E_{c} \subseteq E^{\prime}=\bigcup_{x \in E} x E_{x}$ and it follows that $E^{\prime} \in \mathcal{P}_{C}=\mathscr{P}_{C V}$. Therefore, $\mathscr{P}_{C V}$ is special by Lemma 3.9.

(b) Since $\mathscr{P}_{B}(S)=\{S\}, \mathscr{P}_{B}$ is special iff $S=\bigcup_{s \in S} s S=S^{2}$.

(c) Let $D \in \mathscr{P}_{D}(S)$ and for each $x \in D$ let $D_{x} \in \mathscr{P}_{D}(S)$. By Lemma 3.9

and Lemma 3.1, it will be sufficient to show that $D^{\prime}=\bigcup_{x \in D} x D_{x} \in \mathcal{P}_{D}\left(D_{S}\right)$. Let $0 \neq m, n \in D$. By the hypothesis there exists $s \in S$ such that $m s \neq 0$. Since $D_{n} \in \mathcal{P}_{D}(S)$, there exists $s^{\prime} \in S^{1}$ such that $m s s^{\prime} \neq 0$ and $s s^{\prime} \in D_{n}$. Thus, $m\left(s s^{\prime}\right) \neq 0$ and $n\left(s s^{\prime}\right) \in n D_{n} \subseteq D^{\prime}$. Therefore, $D^{\prime} \in \mathcal{P}_{D}\left(D_{s}\right)$.

(d) Let $0 \neq m \in S$ such that $m S=0$. Then for $D \in \mathcal{P}_{D}(S)$ and $s \in S$ there exists $s^{\prime} \in S^{1}$ such that $0 \neq m s^{\prime}$ and $s s^{\prime} \in D$. However, since $m S=0$ we must have $s^{\prime}=$ 1 and it follows that $D=S$. Therefore, $\mathcal{P}_{D}(S)=\{S\}=\mathcal{P}_{B}(S)$ in this case.

(e) Let $D \in \mathscr{P}_{S D}(S)$ and for each $x \in D$ let $D_{x} \in \mathscr{P}_{S D}(S)$. As in (c), it is suf-

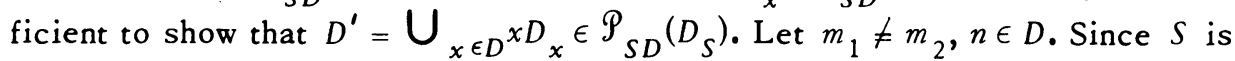
right reductive there exists $s \in S$ such that $m_{1} s \neq m_{2} s$. Hence since $D_{n} \in \mathcal{P}_{S D}(S)$, there exists $s^{\prime} \in S^{1}$ such that $m_{1} s s^{\prime} \not m_{2} s s^{\prime}$ and $s s^{\prime} \in D_{n}$. Therefore, $m_{1}\left(s s^{\prime}\right) \neq$ $m_{2}\left(s s^{\prime}\right)$ and $n\left(s s^{\prime}\right) \in n D_{n} \subseteq D^{\prime}$ and we have $D^{\prime} \in \mathcal{P}_{D}\left(D_{S}\right)$.

(f) Let $m_{1} \neq m_{2} \in S$ such that $m_{1} S=m_{2} S$ and let $D \in \mathcal{P}_{S D}(S)$. Then for $s \in$ $S$, there exists $s^{\prime} \in S^{1}$ such that $m_{1} s^{\prime} \neq m_{2} s^{\prime}$ and $s s^{\prime} \in D$. However, $s^{\prime}$ must be 1 . Therefore $D=S$ and we have $\mathscr{P}_{S D}(S)=\{S\}=\mathscr{P}_{B}(S)$.

We will now return to the general problem of extending a right quotient filter $\mathcal{P}$ on $S$ to a right quotient filter on any $\mathcal{P}$-quotient semigroup over $S$.

Theorem 3.12. Let $\mathfrak{P}^{\prime}=\mathcal{P}^{\prime}(S)$ be a base for a special right quotient filter

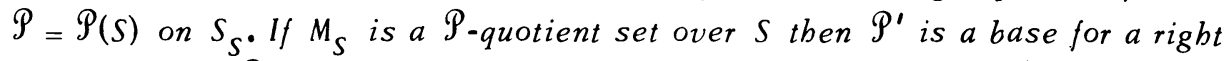
quotient filter $\mathcal{P}\left(M_{S}\right)$ which is independent of the base taken for $\mathscr{P}(S)$.

Proof. Let $A, B \in \mathcal{P}^{\prime}$ and let $f \in \operatorname{Hom}_{S}(A, M)$. By Proposition 3.5, it is sufficient to show that $f^{-1}(B)$ contains an element of $\mathcal{P}^{\prime}$. For each $a \in A$, let $E_{\boldsymbol{a}}=$ $[f(a)]^{-1} S$. Then since $M_{S}$ is a $\mathcal{P}$-quotient set over $S, E_{a} \in \mathcal{P}$. Note that for each element $s \in E_{a}, f(a s)=f(a)_{s} \in S$. Let $E^{\prime}=\bigcup_{a \in A} a E_{a}$. Then $E^{\prime} \in \mathcal{P}$ since $\mathcal{P}$ is special. If $f^{\prime}=f \mid E^{\prime}$ then $f^{\prime} \in \operatorname{Hom}_{S}\left(E^{\prime}, S\right)$. Hence $f^{\prime-1}(B) \in \mathcal{P}(S)$ and it follows that there exists $C \in \mathfrak{P}^{\prime}$ such that $C \subseteq f^{-1}(B) \subseteq f^{-1}(B)$. An argument similar to that of Theorem 3.8 shows that $\mathcal{P}\left(M_{S}\right)$ is independent of the base $\mathcal{P}^{\prime}$ taken for $\mathcal{P}(S)$.

Theorem 3.13. Let $\mathcal{P}^{\prime}=\mathcal{P}^{\prime}(S)$ be a base for a special right quotient filter $\mathcal{P}(S)$ on $S_{S}$. Let $T$ be a $\mathcal{P}(S)$-quotient semigroup over $S$. Then $\mathcal{P}^{\prime}(T)=\left\{E T^{1}\right.$ : $\left.E \in \mathcal{P}^{\prime}\right\}$ is a base for a special right quotient filter $\mathcal{P}(T)$ on $T_{T}$ which is independent of the base $\mathfrak{P}^{\prime}$ taken for $\mathcal{P}(S)$.

Proof. By Theorem 3.12 and Theorem 3.8, $\mathfrak{P}^{\prime}(T)$ is a base for a right quo- 
tient filter $\mathcal{P}(T)$ on $T_{T}$ which is independent of the base $\mathcal{P}^{\prime}$ taken for $\mathcal{P}(S)$. Hence we need only show that $\mathscr{P}(T)$ is special. Without loss of generality we may assume that $\mathfrak{P}^{\prime}=\mathscr{P}(S)$. Let $D \in \mathscr{P}(T)$ and let $D_{x} \in \mathcal{P}(T)$ for each $x \in D$. There exists $E \epsilon$ $\mathcal{P}(S)$ such that $E \subseteq E T^{1} \subseteq D$ and, for each $e \in E$, there exists $E_{e} \in \mathcal{P}(S)$ such that $E_{e} \subseteq E_{e} T^{1} \subseteq D_{e}$. Let $E^{\prime}=\bigcup_{e \in E} e E_{e}$. Then $E^{\prime} \in \mathcal{P}(S)$ and we have $E^{\prime} T^{1}=$ $\left(\bigcup_{e \in E} e E_{e}\right) T^{1} \subseteq\left(\bigcup_{x \in D} x D_{x}\right) T^{1} \subseteq \bigcup_{x \in D} x D_{x}$. Therefore, $\bigcup_{x \in D} x D_{x} \in \mathcal{P}(T)$ and the theorem follows.

The class $\mathcal{P}(T)$ will be called the special right quotient filter induced by $\mathcal{P}(S)$ on $T_{T}$.

The next lemma gives a general analogue of the transitivity property given by Lemma 3.1.

Lemma 3.1.4. Let $\mathcal{P}(S)$ be a special right quotient filter on $S$. Let $T$ be a $\mathcal{P}(S)$-quotient semigroup over $S$ and let $\mathcal{P}(T)$ be the special right quotient filter on $T_{T}$ induced by $\mathscr{P}(S)$. Let $U_{T}$ be a $\mathcal{P}(T)$-quotient $T$-set over $T$. Then $U_{S}$ is a $\mathcal{P}(S)$-quotient $S$-set over $S$.

Proof. Let $u \in U$. We need to show that $\left(u^{-1} S\right)_{S} \in \mathcal{P}(S)$. Since $U_{T}$ is a $\mathcal{P}(T)$ quotient $T$-set over $T,\left(u^{-1} T\right)_{T} \in \mathcal{P}(T)$ and, since $\mathscr{P}(T)$ is induced by $\mathscr{P}(S)$, there exists $E \in \mathscr{P}(S)$ such that $E \subseteq E T^{1} \subseteq\left(u^{-1} T\right)_{T}$. For every $s \in E$, let $E_{s}=$ $\left[(u s)^{-1} S\right]_{S}$. Then $E_{s} \in \mathcal{P}(S)$ for all $s \in E$, since $u s \in T$ and $T$ is a $\mathcal{P}(S)$-quotient semigroup over $S$. Let $E^{\prime}=\bigcup_{s \in E} s E_{s}$. Then $E^{\prime} \in \mathcal{P}(S)$ and $E^{\prime} \subseteq\left(u^{-1} s\right)_{s}$. Therefore, $\left(u^{-1} s\right)_{s} \in \mathcal{P}(S)$.

4. The torsion congruence induced by a right quotient filter. A generalization of the singular congruence and the torsion relation defined by Feller and Gantos [6] will now be given. We begin by defining the $\mathfrak{P}_{\text {-torsion relation on } M_{S} \text { and }}$ noting that it is an $S$-congruence when $\mathcal{P}$ is an R.Q.F. Several facts concerning the $\mathcal{P}_{\text {-torsion congruence when }} \mathcal{P}$ is one of the standard filters are then given.

In general, let $\mathcal{P}$ be any class of right ideals of $S$ and let $M_{S}$ be a right $S$ set. Let $\psi_{\boldsymbol{g}}\left(M_{S}\right)=\left\{\left(m_{1}, m_{2}\right) \in M \times M: m_{1} P=m_{2} P\right.$ for some $\left.P \in \mathcal{P}\right\}$. Then $\psi_{\boldsymbol{g}}\left(M_{S}\right)$

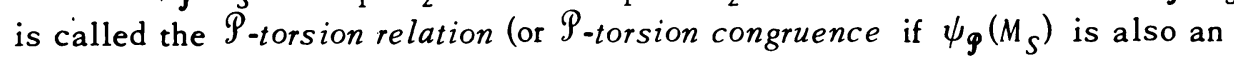
$S$-congruence). When $\mathcal{P}$ is an R.Q.F. on $S_{S}$ this relation is indeed an $S$-congruence as shown in the following lemma which is easily proved using the properties of a right quotient filter.

Lemma 4.1. Let $\mathfrak{P}^{\prime}$ be any base for a right quotient filter $\mathscr{P}$ on $S_{S}$. Then

(a) $\psi_{g^{\prime}}\left(M_{S}\right)=\psi_{\varphi}\left(M_{S}\right)$;

(b) $\psi_{\varphi}\left(M_{S}\right)$ is an $S$-congruence on $M_{S}$; and

(c) if $M=S$ then $\psi_{\boldsymbol{g}}\left(S_{S}\right)$ is a 2-sided congruence on $S$. 
If $\mathfrak{P}=\mathscr{P}_{I}, \mathscr{P}_{D}, \mathscr{P}_{S D}, \mathscr{P}_{C V}$, or $\mathfrak{P}_{B}$ then the corresponding $\mathscr{P}_{\text {-torsion congruence }}$ will be denoted by $\psi_{I}=\psi_{I}\left(M_{S}\right), \psi_{D}=\psi_{D}\left(M_{S}\right), \psi_{S D}=\psi_{S D}\left(M_{S}\right), \psi_{C V}=\psi_{C V}\left(M_{S}\right)$, or $\psi_{B}=\psi_{B}\left(M_{S}\right)$ respectively. In case $M=S$ the subscript $S$ will be omitted. The congruence $\psi_{I}\left(M_{S}\right)$ is generally known as the singular congruence on $M_{S}$.

The following lemma shows that the order relation " $\subseteq$ " is preserved in passing from right quotient filters to $\mathcal{P}_{\text {-torsion congruences. }}$

Lemma 4.2. Let $\mathcal{P}_{1}$ and $\mathcal{P}_{2}$ be any two right quotient filters on $S_{S}$. If $\mathcal{P}_{1} \subseteq$ $\mathcal{P}_{2}$ then $\psi_{\boldsymbol{\Phi}_{1}} \subseteq \psi_{\boldsymbol{\Phi}_{2}}$. In particular, we bave $\psi_{B} \subseteq \psi_{S D} \subseteq \psi_{D} \subseteq \psi_{I}$ and $\psi_{C V} \subseteq \psi_{I}$.

Let $S$ be a semigroup with right cancellable elements and let $C$ be a subsemigroup of $S$ consisting entirely of right cancellable elements of $S$ such that $S$ has the right common multiple property with respect to $C$. In Proposition 3.10, it was shown that $\mathscr{P}_{C V}=\left\{R_{S} \subseteq S_{S}: R \cap C \neq \square\right\}=\mathscr{P}_{C}$. The next lemma shows that $\psi_{C V}\left(M_{S}\right)$ is the same as the torsion relation defined by Feller and Gantos in [6].

Lemma 4.3. Let $C$ be as above and let $S$ have the R.C.M.P. with respect to C. Then $\psi_{C V}\left(M_{S}\right)=\left\{\left(m_{1}, m_{2}\right): m_{1} c=m_{2} c\right.$ for some $\left.c \in C\right\}$.

Proof. Let $\left(m_{1}, m_{2}\right) \in \psi_{C V}\left(M_{S}\right)$. Then there exists $E \in P_{C V}$ such that $m_{1} E=$ $m_{2} E$. Since $E \cap C \neq \square$, it follows that $\left(m_{1}, m_{2}\right)$ is a member of the right side. Now, let $\left(m_{1}, m_{2}\right)$ be an element of the right side. Then there exists $c \in C$ such that $m_{1} c=m_{2} c$. Let $E=c S$. Then since $c^{2} \in c S \cap C$ we have $c S \in \mathcal{P}_{C V}$. Clearly, $m_{1} E \div m_{2} E$ and we have $\left(m_{1}, m_{2}\right) \in \psi_{C V}\left(M_{S}\right)$.

The following corollary is evident from the lemma.

Corollary 4.4. Under the hypotheses of Lemma $4.3 \psi_{C V}(S)=\iota$.

It is clear from the definitions that $\mathcal{P}_{D}\left(M_{S}\right)$ is always contained in $\mathcal{P}_{I}\left(M_{S}\right)$. The next lemma gives a sufficient condition for equality of the se two filters.

Lemma 4.5. If $\psi_{I}\left(M_{S}\right)$ is 0-restricted then $\mathcal{P}_{I}\left(M_{S}\right)=\mathscr{P}_{D}\left(M_{S}\right)$.

Proof. We need only show that: $\mathscr{P}_{I}\left(M_{S}\right) \subseteq \mathcal{P}_{D}\left(M_{S}\right)$. Let $N_{S} \in \mathscr{P}_{I}\left(M_{S}\right)$ and let $0 \neq m, n \in M$. In a similar manner to the note following Lemma 3.2 we have $n^{-1} N=$ $\{s \in S: n s \in N\} \in \mathcal{P}_{I}(S)$. If $m\left(n^{-1} N\right)=0$ then $(m, 0) \in \psi_{I}\left(M_{S}\right)$. However, $m \neq 0$ and $\psi_{I}\left(M_{S}\right)$ is 0 -restricted. Therefore, $m\left(n^{-1} N\right) \neq 0$ and it follows that there exists $s \in S^{1}$ such that $m s \neq 0$ and $n s \in N$. Hence, $N_{S} \in \mathscr{P}_{D}\left(M_{S}\right)$.

This lemma and the lemma which follows show that "dense" and "intersection large" are the same concepts on a regular semigroup.

Lemma 4.6. If $S \subseteq T$ such that $S \in \mathcal{P}_{I}\left(T_{S}\right)$ and $T$ is a regular semigroup, then $\psi_{I}\left(T_{S}\right)$ is 0-restricted.

Proof. Let $E \in \mathcal{P}_{I}(S)$ and let $0 \neq t \in T$. Let $t^{\prime}$ be an inverse of $t$ in $T$. Since 
$S \in \mathcal{P}_{I}\left(T_{S}\right)$ we have $E \in \mathcal{P}_{I}\left(T_{S}\right)$. Thus there exists $s \in S^{1}$ such that $0 \neq t^{\prime} s \in E$. Hence $0 \neq t^{\prime} s=t^{\prime} t t^{\prime} s \in E$ and it follows that $0 \neq t\left(t^{\prime} s\right) \in t E$. Therefore, $\psi_{I}\left(T_{s}\right)$ must be 0 -restricted since $E$ is an arbitrary element of $\mathcal{P}_{I}\left(T_{S}\right)$ and contains an element $x$ such that $t x \neq 0$ for each $0 \neq t \in T$.

In general, $\mathcal{P}_{I}(S)$ is not a special right quotient filter. However, the next proposition gives necessary and sufficient conditions for this to be true.

Proposition 4.7. The following are equivalent:

(a) $\mathcal{P}_{l}(S)$ is special.

(b) $\psi_{I}(S)$ is 0-restricted.

(c) $\mathscr{P}_{I}(S)=\mathscr{P}_{D}(S)$ and $\{s \in S: s S=0\}=0$.

Proof. (a) $\Rightarrow(\mathrm{b})$. Let $A=\left\{s \in S:(s, 0) \in \psi_{I}(S)\right\}$. We need to show that $A=0$. By Zorn's lemma there exists a maximal sub $S$-set $A_{S}^{\prime}$ of $S$ such that $A \cap A^{\prime}=0$. It easily follows that $B=A \cup A^{\prime} \in \mathcal{P}_{I}(S)$. For each $b \in B$, let

$$
D_{b}= \begin{cases}b^{-1}\{0\} & \text { if } b \in A, \\ B & \text { if } b \in A^{\prime} .\end{cases}
$$

Note that $b^{-1}\{0\} \in \mathscr{P}_{I}(S)$ for each $b \in A$. Hence $D=\bigcup_{b \in B} b D_{b} \in \mathscr{P}_{I}(S)$ since $\mathcal{P}_{I}(S)$ is special. However, we can write

$$
D=\left[\bigcup_{b \in A} b\left(b^{-1}\{0\}\right)\right] \cup\left[\bigcup_{b \in A^{\prime}}, b B\right]=\bigcup_{b \in A^{\prime}} b B \subseteq A^{\prime} .
$$

Thus it follows that $A^{\prime} \in \mathcal{P}_{I}(S)$ and since $A \cap A^{\prime}=0$ we must have $A=0$.

(b) $\Rightarrow(\mathrm{c})$. This follows immediately from Lemma 4.5 .

(c) $\Rightarrow(a)$. This is essentially a restatement of Proposition 3.11(c).

Now let $\mathcal{P}$ denote one of $\mathcal{P}_{I}, \mathcal{P}_{D}$, or $\mathcal{P}_{S D}$. If $S$ and $T$ are semigroups such that $S \subseteq T$ then $T$ has a $\mathcal{P}(S)$-torsion congruence as well as a $\mathcal{P}(T)$-torsion congruence. These are the same under the conditions given below.

Theorem 4.8. Let $S$ and $T$ be semigroups such that $S \in \mathcal{P}_{D}\left(T_{S}\right)$. Then $\psi_{D}\left(T_{S}\right)=\psi_{D}(T)$ and $\psi_{I}\left(T_{S}\right)=\psi_{I}(T)$. Also, if $S \in \mathcal{P}_{S D}\left(T_{S}\right)$ then $\psi_{S D}\left(T_{S}\right)=$ $\psi_{S D}(T)$.

Proof. Let $\mathcal{P}$ denote any of $\mathcal{P}_{D}, \mathcal{P}_{S D}$, or $\mathcal{P}_{I}$ and let $\psi$ denote the corresponding torsion congruence. If $\left(t_{1}, t_{2}\right) \in \psi(T)$ then there exists $E \in \mathcal{P}(T)$ such that $t_{1} E=t_{2} E$. By Theorem $3.7((\mathrm{a}),(\mathrm{b})$, or (c) depending on the choice of $\mathcal{P}$ ), there exists $E^{\prime} \in \mathscr{P}(S)$ such that $E^{\prime} \subseteq E^{\prime} T^{1} \subseteq E$. Thus $t_{1} E^{\prime}=t_{2} E^{\prime}$ and we have $\left(t_{1}, t_{2}\right) \in \psi_{\rho}\left(T_{S}\right)$. On the other hand if $\left(t_{1}, t_{2}\right) \in \psi_{\rho}\left(T_{S}\right)$, there exists $E \in \mathcal{P}(S)$ such that $t_{1} E=t_{2} E$. Hence $t_{1}\left(E T^{1}\right)=t_{2}\left(E T^{1}\right)$ and $E T^{1} \in \mathcal{P}(T)$ by the same 
theorem mentioned above. Therefore, $\left(t_{1}, t_{2}\right) \in \psi(T)$ and the result follows.

The theorem can also be stated for any special filter and for any $\mathcal{P}$-quotient semigroup. The proof is similar to that of the previous theorem.

Theorem 4.9. If $\mathcal{P}=\mathscr{P}(S)$ is a special right quotient filter on $S$ and $T$ is a

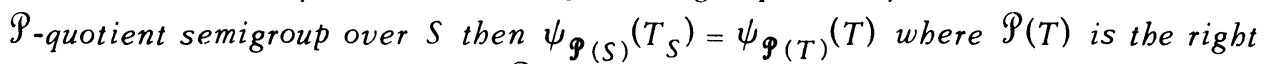
quotient filter induced on $T$ by $\mathcal{P}$.

Let $\mathcal{P}$ be an R.Q.F. on $S$. The $S$-set $M_{S}$ will be called $\mathcal{P}$-torsion free if $\psi_{\varphi}\left(M_{S}\right)=\iota$. We have already seen that if $S$ has a subsemigroup $C$ of right cancellable elements and has the R.C.M.P. with respect to $C$ then $S$ is $\mathscr{P}_{C V}(S)$-torsion free. We observe below that $S$ is $\mathcal{P}_{S D^{\text {ttorsion }} \text { free for a large class of semigroups }}$ which includes each semigroup with ident ity.

Lemma 4.10. For all semigroups $S, \psi_{S D}(S)=\psi_{B}(S)$. Hence $\psi_{S D}(S)$ is the identity congruence iff $S$ is right reductive.

Proof. Clearly, $\psi_{B}(S)=\iota$ iff $S$ is right reductive. Suppose $(s, t) \in \psi_{S D}(S)$ such that $s \neq t$. Then there exists $D \in \mathcal{P}_{S D}(S)$ such that $s D=t D$. If $s^{\prime} \in S$ such that $s s^{\prime} \neq t s^{\prime}$ then there exists $t^{\prime} \in S^{1}$ such that $s s^{\prime} t^{\prime} \neq t s^{\prime} t^{\prime}$ and $s^{\prime} t^{\prime} \in D$, which is a contradiction. Thus $s S=t S$ and it follows that $\psi_{B}(S)=\psi_{S D}(S)$.

McMorris showed in [12] that $\mathcal{P}_{I}(S)=\mathscr{P}_{S D}(S)$ when $\psi_{I}(S)=\imath$. A similar proof gives this result for all $S$-sets.

Lemma 4.11. If $\psi_{I}\left(M_{S}\right)=\imath$ then $\mathscr{P}_{S D}\left(M_{S}\right)=\mathscr{P}_{D}\left(M_{S}\right)=\mathscr{P}_{I}\left(M_{S}\right)$.

We also have the following two lemmas involving $\mathcal{P}$-quotient sets.

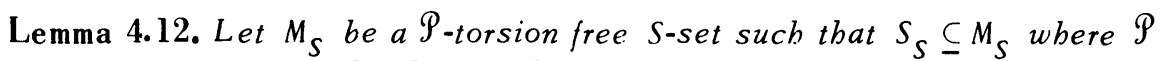

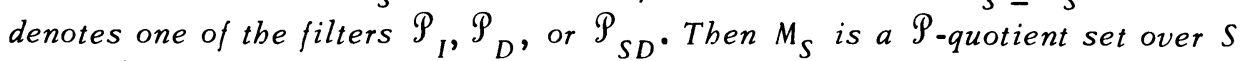
iff $S \in \mathcal{P}\left(M_{S}\right)$.

Proof. If $S \in \mathcal{P}\left(M_{S}\right)$ then $M_{S}$ is a $\mathcal{P}$-quotient set over $S$ by Proposition 3.6.

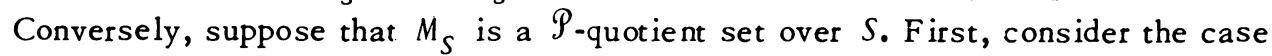
where $\mathcal{P}=\mathscr{P}_{D}$ and let $0 \neq m, n \in M$. Then $n^{-1} S \in \mathcal{P}(S)$ and $m\left(n^{-1} S\right) \neq 0$ since $\psi_{D}\left(M_{S}\right)=\iota$. Thus there exists $s \in S$ such that $0 \neq m s$ and $n s \in S$ and it follows that $S \in \mathcal{P}_{D}\left(M_{S}\right)$. Next, suppose that $\mathscr{P}=\mathscr{P}_{I}$. Since $\psi_{I}\left(M_{S}\right)=\iota$, we have $\mathscr{P}_{I}\left(M_{S}\right)=$ $\mathscr{P}_{D}\left(M_{S}\right)$ by Lemma 4.11 and it also follows that $\psi_{I}(S)=\imath$. Thus $\mathscr{P}_{I}(S)=\mathscr{P}_{D}(S)$ by Lemma 4.11 again and the result follows from the previous case. The proof for the case $\mathcal{P}=\mathcal{P}_{S D}$ is similar to that of the first case and will be omitted.

Lemma 4.13. Let $\mathcal{P}$ be an arbitrary right quotient filter on $S$ such that $S$ is

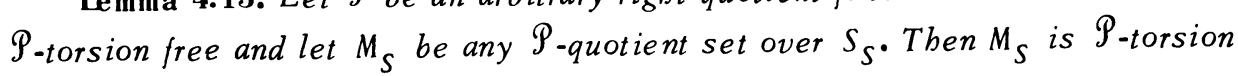
free iff $S_{S}$ is large in $M_{S}$. 
Proof. ( $\Rightarrow$ ) Let $\Phi \in \operatorname{Hom}_{S}(M, K)$ such that $\Phi \mid S$ is 1-1 where $K_{S}$ is an arbitrary $S$-set. Let $m_{1}, m_{2} \in M$ such that $\Phi\left(m_{1}\right)=\Phi\left(m_{2}\right)$. Let $D=m_{1}^{-1} S \cap m_{2}^{-1} S \epsilon$ $\mathcal{P}$. Since $\Phi\left(m_{1} s\right)=\Phi\left(m_{2} s\right)$ for all $s \in D$ and since $\Phi \mid S$ is $1-1$, we have $m_{1} D$ \%

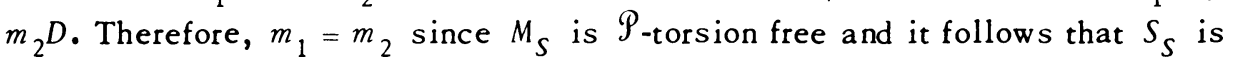
large in $M_{S}$.

$(\Leftarrow)$ Since $\psi_{\boldsymbol{\rho}}(S)=\psi_{\boldsymbol{\rho}}\left(M_{S}\right) \mid S=\iota$, it follows from Lemma 1.1 that $\psi_{\boldsymbol{\rho}}\left(M_{S}\right)=\imath$.

5. Right quotient semigroups. Let $S$ be a semigroup, $M_{S}$ be a right $S$-set and $\mathcal{P}$ be a right quotient filter on $M_{S}$. Let $F=F_{\boldsymbol{\rho}}\left(M_{S}\right)=\bigcup_{D \in \rho} \operatorname{Hom}_{S}(D, M)$ and define multiplication on $F$ by $f g=b$ where $b: D_{g} \cap g^{-1}\left(D_{f}\right) \rightarrow M$ is given by $b(x)=f(g(x))$. Then under this multiplication $F$ is a semigroup, called the semigroup of partial S-homomorphisms of $M_{S}$ with respect to $\mathcal{P}$.

Lemma 5.1. Define a binary relation $\omega=\omega_{\rho}\left(M_{S}\right)$ on the semigroup $F$ by $(f, g) \in \omega$ iff there exists $D \in \mathcal{P}$ such that $f|D=g| D$. Then $\omega$ is a 2-sided congruence on $F$.

The semigroup $Q_{\varphi}=Q_{\varphi}\left(M_{S}\right)=F / \omega$ will be called the semigroup of right quotients of $M_{S}$ with respect to $\mathcal{P}$. The elements of $Q_{\boldsymbol{g}}\left(M_{S}\right)$ will be denoted by $\bar{f}$ where $f \in F$. When $\mathcal{P}_{=} \mathcal{P}_{I}, \mathcal{P}_{D}, \mathcal{P}_{S D}, \mathcal{P}_{C V}$, or $\mathcal{P}_{B}$ then $Q_{\mathcal{P}}$ will be denoted by $Q_{I}, Q_{D}, Q_{S D}, Q_{C V}$, or $Q_{B}$ respectively. As before, the subscript $S$ in $Q_{\mathcal{P}}\left(M_{S}\right)$ will be omitted when $M=S$.

Note that $Q_{B}\left(M_{S}\right)=\operatorname{Hom}_{S}(M, M)$. More generally, the following lemma shows that if the R.Q.F. $P$ on $M_{S}$ has a least element $D_{S}$ under set inclusion then there is a natural isomorphism between $Q_{\varphi}\left(M_{S}\right)$ and $\operatorname{Hom}_{S}(D, D)$.

Lemma 5.2. Let $\mathcal{P}$ be a right quotient filter on $M_{S}$ with a least element $D_{S}$. Then $Q_{\mathscr{\rho}}\left(M_{S}\right) \approx \operatorname{Hom}_{S}(D, D)$ under the map $f \rightarrow \bar{f}$ where $f \in \operatorname{Hom}_{S}(D, D)$ and multiplication on $\operatorname{Hom}_{S}(D, D)$ is composition.

Proof. The isomorphism will clearly follow once it is shown that $\operatorname{Hom}_{S}(D, M)=$ $\operatorname{Hom}_{S}(D, D)$. Let $f \in \operatorname{Hom}_{S}(D, M)$. Then $f^{-1}(D) \in \mathcal{P}$ and since $D$ is the least element of $\mathcal{P}$ we have $D \subseteq f^{-1}(D)$. Thus, $D=f^{-1}(D)$ and it follows that $f(D) \subseteq D$. Therefore, $f \in \operatorname{Hom}_{S}(D, D)$. Since the reverse inclusion is obvious, we have equality.

In this paper we are mainly concerned with the case where $M$ is the semigroup $S$. As noted below we see that there is a natural representation of $S$ in $Q_{\rho}(S)$.

Let $\mathcal{P}$ be a right quotient filter on $S_{S}$. For each $s \in S$, define $\phi_{s}: S \rightarrow S$ by $\phi_{s}(t)=s t$. Then $\underline{\phi}_{s} \in \operatorname{Hom}_{S}(S, S)$. It is easily seen that the mapping $\phi: S \rightarrow Q=$ $Q_{\varphi}(S)$ by $\phi(s)=\bar{\phi}_{s}$ is a representation of $S$ in $Q_{\rho}(S)$. The image of $S$ under $\phi$ will be denoted by $\bar{S}$. Since $\bar{S}$ is a subsemigroup of $Q, Q$ may be regarded as a 
centered right $\bar{S}$-set $Q_{\bar{S}}$ in a natural way. Also, $Q$ becomes a centered right $S$ set $Q_{S}$ by defining $\bar{f} s=\bar{f}_{s}$ for each $\bar{f} \in Q$ and for each $s \in S$. It is easy to show that the following lemmas involving the representation of $S$ in $Q_{\mathcal{P}}(S)$ are valid.

Lemma 5.3. $\psi_{\varphi}(s)=\phi^{-1} \circ \phi$.

Lemma 5.4. For each $f \in F_{\rho}(s)$ and for each $s \in D_{f}, f \phi_{s}=\phi_{f(s)}$.

When $\psi_{\boldsymbol{\rho}}(S)=\imath$, we shall assume that $S$ is embedded in $Q=Q_{\boldsymbol{g}}(S)$ under the identification $s \leftrightarrow \bar{\phi}_{s}$. From Lemma 5.4 we see that $\bar{f} s=f(s)$ for each $\bar{f} \in Q_{g}(s)$ and for each $s \in D_{f}$ under the identification described above. Furthermore, since $D_{f} \subseteq(\bar{f})^{-1} S$ and $D_{f} \in \mathcal{P}, Q_{\varphi}(S)$ is also a $\mathcal{P}$-quotient semigroup over $S$. In addition, the next lemma shows that $Q_{S}$ is $\mathcal{P}(S)$-torsion free.

Lemma 5.5. Let $\mathcal{P}=\mathscr{P}(S)$ be a right quotient filter on $S$ and let $Q=Q_{\varphi}(S)$. If $S$ is $\mathcal{P}_{\text {-torsion free then }} Q_{S}$ is $\mathcal{P}_{\text {-torsion free. }}$

Proof. Let $\left(\bar{f}_{1}, \bar{f}_{2}\right) \in \psi_{\mathcal{P}}\left(Q_{S}\right)$. Then there exists $E \in \mathcal{P}$ such that $\bar{f}_{1} E=\bar{f}_{2} E$. Let $E^{\prime}=E \cap D_{f_{1}} \cap D_{f_{2}} \in \mathcal{P}$. Then for each $s \in E^{\prime}$, we have $f_{1}(s)=\bar{f}_{1} s=\bar{f}_{2} s=$ $f_{2}(s)$ and it follows that $\bar{f}_{1}=\bar{f}_{2}$.

The following corollary is immediate from Lemma 4.13 and the remarks preceding the above theorem.

Corollary 5.6. Let $\mathcal{P}$ be a right quotient filter on $S$ and let $Q=Q_{\mathcal{P}}(S)$. If $S$

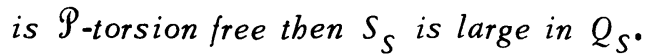

The next theorem shows among other things that each $\mathcal{P}_{\text {-torsion free }} \mathcal{P}_{\text {-quo- }}$ tient set (semigroup) can be embedded in $Q_{\mathscr{\rho}}(S)$ as an $S$-set (semigroup) such that the elements of $S$ are fixed under the identification $s \leftrightarrow \bar{\phi}_{S}$.

Theorem 5.7. Let $M_{S}$ be a $\mathcal{P}$-quotient set (semigroup) over $S$. Then there exists an S-bomomorphism (bomomorphism) $\Phi: M_{S} \rightarrow Q_{\mathcal{P}}(S)=Q_{S}$ such that

(a) $\Phi(s)=\bar{\phi}_{s}$ for each $s \in S$ and

(b) $\Phi^{-1} \circ \Phi=\psi_{\varphi}\left(M_{S}\right)$.

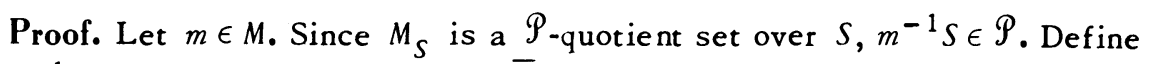
$\underline{\theta}_{m}: m^{-1} S \rightarrow S$ by $\theta_{m}(s)=m s$. Then $\bar{\theta}_{m} \in Q=Q_{\mathscr{\rho}}(S)$. Let $\Phi: M \rightarrow Q$ by $\Phi(m)=$ $\bar{\theta}_{m}$. For each $s \in S$, we have $D_{\theta_{s}}=s^{-1} S=S$. Thus $\Phi(s)=\bar{\theta}_{s}=\bar{\phi}_{s}$ and (a) follows. Furthermore, $\Phi\left(m_{1}\right)=\Phi\left(m_{2}\right)$ iff $\bar{\theta}_{m_{1}}=\bar{\theta}_{m_{2}}$ which is true iff there exists $E \epsilon$ $\mathcal{P}$ such that $m_{1} s=\theta_{m_{1}}(s)=\theta_{m_{2}}(s)=m_{2} s$ for all $s \in E$. Hence it follows that $\Phi^{-1} \circ \Phi=\psi_{\rho}\left(M_{s}\right)$. Now let $m \in M$ and $s \in S$. Then $\Phi(m s)=\bar{\theta}_{m s}$ and $\Phi(m) s=$ $\bar{\theta}_{m} s=\bar{\theta}_{m} \bar{\phi}_{s}=\bar{\theta}_{m} \phi_{s}$. Let $t \in D_{\theta_{m s}} \cap D_{\theta_{m} \theta_{s}} \in \mathcal{P}$. Then $\theta_{m s}(t)=(m s) t=m(s t)=$ $\theta_{m}(s t)=\theta_{m} \phi_{s}(t)$. Thus it follows that $\Phi$ is an $S$-homomorphism. Now suppose $M$

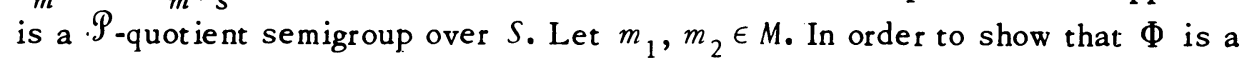


semigroup homomorphism it is sufficient to show that $\bar{\theta}_{m_{1} m_{2}}=\bar{\theta}_{m_{1}} \bar{\theta}_{m_{2}}$. Let $t \epsilon$ $D_{\theta_{m_{1} m_{2}}} \cap D_{\theta_{m_{1}} \theta_{m_{2}}} \in \mathcal{P}$. Then $\theta_{m_{1} m_{2}}(t)=\left(m_{1} m_{2}\right) t=m_{1}\left(m_{2} t\right)=\theta_{m_{1}} \theta_{m_{2}}(t)$. Therefore, $\bar{\theta}_{m_{1} m_{2}}=\bar{\theta}_{m_{1}} \bar{\theta}_{m_{2}}$.

As the following corollary shows, $Q_{\mathcal{P}}(S)$ is the maximal $\mathcal{P}_{\text {-torsion free }} \mathcal{P}$. quotient semigroup over $S$ (up to semigroup isomorphism over $S$ ) when $S$ is $\mathscr{P}_{\text {-tor- }}$ sion free.

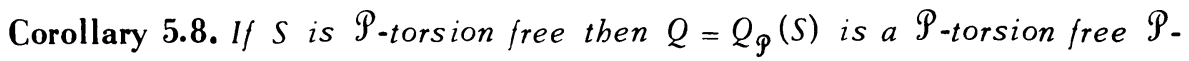
quotient semigroup over $S$ which contains an $S$-isomorphic (isomorphic) copy over $S$ of every $\mathcal{P}_{\text {-torsion free }} \mathcal{P}_{\text {-quotient set }}$ (semigroup) over $S$.

Proof. By Lemma 5.5 and the remarks preceding it, $Q$ is a $\mathcal{P}_{\text {-torsion free }} \mathcal{P}$. quotient semigroup over $S$. Since $\psi_{\mathcal{\rho}}(S)=\imath$, the rest follows from Theorem 5.7.

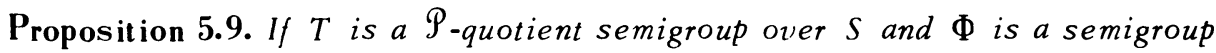
endomorphsim on $T$ such that $\Phi \mid S=\iota_{S}$ then $\Phi \subseteq \psi_{\Phi}\left(T_{S}\right)$.

Proof. Suppose $\Phi\left(t_{1}\right)=t_{2}$. Let $D=t_{1}^{-1} S \cap t_{2}^{-1} S \in \mathcal{P}$. Then for each $s \in D$, we have $t_{1} s=\Phi\left(t_{1} s\right)=\Phi\left(t_{1}\right) \Phi(s)=t_{2} s$. Thus, it follows that $\left(t_{1}, t_{2}\right) \in \psi_{\varphi}\left(T_{s}\right)$.

Corollary 5.10. If $T$ is a $\mathfrak{P}_{\text {-torsion free }} \mathfrak{P}_{\text {-quotient semigroup over } S \text { then }}$ the only semigroup endomorphism on $T$ fixing $S$ is the identity.

Let $\mathcal{P}=\mathscr{P}(S)$ be a special right quotient filter on $S$ such that $S$ is $\mathcal{P}_{\text {-torsion }}$ free. Let $\mathcal{P}(Q)$ be the special right quotient filter on $Q=Q_{\varphi}(S)$ regarded as a $Q$ set as given by Theorem 3.13. By Lemma 5.5, $Q_{S}$ is $\mathcal{P}(S)$-torsion free and it follows from Theorem 4.9 that $Q_{n}$ is also $\mathcal{P}(Q)$-torsion free. Thus there exists a natural embedding of $Q$ in $Q^{\prime}=Q_{\mathcal{P}(Q)}(Q)$ and under this embedding $Q_{Q}^{\prime}$ is a $\mathcal{P}(Q)$-quotient semigroup over $Q$. The next theorem shows that this embedding is actually onto $Q^{\prime}$.

Theorem 5.11. Let $\mathcal{P}=\mathcal{P}(S)$ be a special right quotient filter on $S$ such that

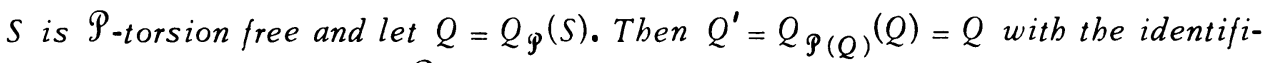
cation given above where $\mathcal{P}(Q)$ is the special right quotient filter induced on $Q_{Q}$ by $\mathcal{P}(S)$.

Proof. By Lemma 5.5 with $S$ replaced by $Q$, we have $Q_{Q}^{\prime}$ is $\mathcal{P}(Q)$-torsion free. We claim that $Q_{S}^{\prime}$ is $\mathcal{P}(S)$-torsion free. Let $\left(t_{1}, t_{2}\right) \in \psi_{\mathcal{Q}(S)}\left(Q_{S}^{\prime}\right)$. Then there exists $E \in \mathcal{P}(S)$ such that $t_{1} E=t_{2} E$. Hence $t_{1}(E Q)=t_{2}(E Q)$ and $E Q \in \mathcal{P}(Q)$. Thus $\left(t_{1}, t_{2}\right) \in \psi_{\mathcal{P}(Q)}\left(Q_{Q}^{\prime}\right)=\iota$ and it follows that $Q_{S}^{\prime}$ is $\mathcal{P}(S)$-torsion free. Furthermore, by Lemma $3.14, Q_{S}^{\prime}$ is a $\mathcal{P}(S)$-quotient semigroup over $S$. So, by Corollary 5.8 there exists a semigroup isomorphism $\Phi$ of $Q^{\prime}$ into $Q$ which fixes $S$. Let $\Phi^{\prime}=$ $\Phi \mid Q$. Then by Corollary 5.10, $\Phi^{\prime}={ }^{\imath} Q$. Therefore, since $\Phi: Q^{\prime} \rightarrow Q$ is an isomorphism which fixes $Q$ it follows that $Q^{\prime}=Q$. 
From Proposition 3.11 we see that $\mathscr{P}_{D}(S)$ is special whenever $S$ is $\mathcal{P}_{D^{\text {tor- }}}$ sion free. Also, from Lemma 4.10 and Proposition 3.11, $\mathcal{P}_{S D}(S)$ is special whenever $S$ is $P_{S D}$ torsion free. Hence the corollary given below for the se particular cases follows.

Corollary 5.12. If $S$ is $\mathcal{P}_{D}$-torsion free then $Q_{D}\left(Q_{D}(S)\right)=Q_{D}(S)$ and if $S$ is $\mathcal{P}_{S D^{\text {torsion }}}$ free then $Q_{S D}\left(Q_{S D}(S)\right)=Q_{S D}(S)$.

In [8], the author noted that $Q_{I}(S)$ is the injective hull of $S_{S}$ and is self-injective whenever $S$ is $\mathscr{P}_{I}$-torsion free. These results can also be obtained as applications of the preceding the ory.

Theorem 5.13. If $S$ is $\mathcal{P}_{I}$-torsion free then $Q=Q_{I}(S)$ is the injective bull of $S_{S}$

Proof. Since $\psi_{I}(S)=\iota$, we see from Corollary 5.6 that $S_{S}$ is large in $Q_{S}$. Let $M_{S}$ be the injective hull of $S_{S}$. Since $S_{S}$ is large in $M_{S}, S_{S} \in \mathcal{P}_{I}\left(M_{S}\right)$ as shown by Feller and Gantos in [6] and we see that $M_{S}$ is a $\mathcal{P}_{I}$-quotient set over $S$. Also by Lemma $4.13, M_{S}$ is $P_{I}$-torsion free. Thus, from Corollary 5.8 we may assume that $M_{S}$ is chosen such that $S_{S} \subseteq M_{S} \subseteq Q_{S}$. However, since $S_{S}$ is large in $Q_{S}$ and since $M_{S}$ is a maximal essential extension of $S_{S}$ we must have $M_{S}=Q_{S}$ and the result follows.

Theorem 5.14. If $S$ is $\mathcal{P}_{l}$-torsion free then $Q=Q_{I}(S)$ is self-injective.

Proof. By Lemma 5.5 we have $\psi_{I}\left(Q_{S}\right)=\imath$. Since $\mathcal{P}_{I}(S)=\mathscr{P}_{D}(S)$ by Lemma 4.5, $S \in \mathcal{P}_{D}\left(Q_{S}\right)$ by Lemma 4.12. Hence by Theorem 4.8, $\psi_{I}(Q)=\imath$. So, again by Lemma 4.5, $\mathscr{P}_{I}(Q)=\mathscr{P}_{D}(Q)$. Therefore, since $\mathcal{P}_{D}(S)=\mathscr{P}_{I}(S)$ and $\mathscr{P}_{D}(Q)=\mathscr{P}_{I}(Q)$ it follows from Corollary 5.12 that $Q_{I}\left(Q_{I}(S)\right)=Q_{I}(S)$. Thus $Q_{Q}$ is injective by Theorem 5.13 where $S$ is replaced by $Q$ in the theorem.

Let $C$ be a subsemigroup of the 2-sided cancellative elements of $S$. A semigroup $Q$ with identity containing $S$ as a subsemigroup is called a classical semigroup of right quotients of $S$ with respect to $C$ if (a) each element of $C$ has a 2-sided inverse in $Q$, and (b) $Q=\left\{a b^{-1}: a \in S, b \in C\right\}$.

The following theorem is a generalization of a theorem in ring theory proved by Asano in [1]. A classical proof of this theorem was also given by Smith in [14].

Theorem 5.15. $S$ has a classical semigroup of right quotients with respect to $C$ iff $S$ bas the R.C.M.P. with respect to $C$.

Proof. ( $\Rightarrow$ ) Let $S \subseteq Q$ where $Q$ is a classical semigroup of right quotients over $S$ with respect to $C$. Let $n \in C$ and $s \in S$. There exists $s_{1} \in S$ and $n_{1} \in C$ such that $n^{-1} s=s_{1} n_{1}^{-1}$. Hence $s n_{1}=n s_{1}$ and it follows that $s C \cap n S \neq \square$.

$(\Leftarrow)$ If $S$ has the R.C.M.P. with respect to $C$ then $\mathcal{P}_{C V}$ as defined in $\S 3$ 
is a special right quotient filter on $S$ by Proposition 3.11. Also by Corollary 4.4, $S_{S}$ is $P_{C V}$-torsion free. Let $Q=Q_{C V}(S)$. As before we can identify $S$ with the semigroup $\Phi(S)=\bar{S}$ in $Q$. Hence without loss of generality $S \subseteq Q$ under the identification $s \leftrightarrow \bar{\phi}_{s}$. For each $n \in C, \phi_{n-}: S \rightarrow n S$ is 1-1 since $n$ is left cancellative. Hence $\phi_{n}^{-1}: n S \rightarrow S$ exists and $\bar{\phi}_{n} \bar{\phi}_{n}^{-1}=\bar{\phi}_{n}^{-1} \bar{\phi}_{n}=\tau_{s}$. Thus each element of $C$ has a 2-sided inverse in $Q$. Now let $\bar{f} \in Q$ and let $n \in D_{f} \cap C$. Then $\bar{f} n=$ $f(n)$. Therefore $\bar{f}=f(n) n^{-1} \in S C^{-1}$ and the theorem follows.

A semigroup $S^{\prime}$ (without 0 ) is said to be left reversible if $s_{1} S^{\prime} \cap s_{2} S^{\prime} \neq \square$ for every $s_{1}, s_{2} \in S^{\prime}$. The following well-known theorem due to Ore (see Clifford and Preston [4]) now follows as a corollary of Theorem 5.15 .

Corollary 5.16. A cancellative semigroup $S^{\prime}$ (without 0 ) is embeddable in a group of right quotients of $S^{\prime}$ iff $S^{\prime}$ is left reversible.

Proof. Let $S=S^{\prime 0}$ and let $C=S^{\prime}$. If $S^{\prime}$ is left reversible then $S$ has the R.C.M.P. with respect to $C=S^{\prime}$. Hence $S$ has a semigroup of right quotients $Q$ with respect to $S^{\prime}$. The properties of $Q$ immediately yield the fact that $Q$ is a group with 0 adjoined. Let $A^{\prime}=Q \backslash\{0\}$. Then $Q^{\prime}$ is a group of right quotients of $S^{\prime}$. The proof of the converse is easy and will be omitted.

Lemma 5.17. Let $S \subseteq T \subseteq U$ where $T$ and $U$ are classical semigroups of quotients over $S$ with respect to $C$. Then $T=U$.

Theorem 5.18. If $S$ has a classical semigroup of quotients $T$ with respect to $C$ then $T$ is unique up to isomorphism.

Proof. Let $T$ be any classical semigroup of quotients of $S$ with respect to $C$ and let $Q=Q_{C V}(S)$ be the one obtained in Theorem 5.15. If $t \in T$ then $t=$ $s n^{-1}$ for some $s \in S$ and some $n \in C$. Thus $n \in t^{-1} S$ and it readily follows that $t^{-1} S \in \mathcal{P}_{C V}(S)$. Since each element of $C$ has an inverse in $T$, it is immediate from Lemma 4.3 that $T_{S}$ is $P_{C V}$-torsion free. Thus $T$ is $P_{C V}$-torsion free and a ${ }^{\text {P }} \mathrm{CV}^{-}$ quotient semigroup over $S$. Hence by Corollary 5.8, $T$ can be embedded in $Q$ such that $S \subseteq T \subseteq Q$ and the result follows from Lemma 5.17 .

6. Right quotient semigroups of 0-direct unions of semigroups. A right ideal $R$ of a semigroup $S$ is called a null right ideal if $R S=0$. Throughout this section we shall let $\left\{S_{a}\right\}_{a \in \Omega}$ be a collection of 0 -disjoint semigroups each having no nonzero null right ideals. The semigroup $S$ will denote the 0 -direct union of $\left\{S_{\alpha}\right\}_{\alpha \in \Omega}$ (i.e., $S$ is the union of the $S_{\alpha}$ 's and $S_{\alpha} S_{\beta}=0$ for $\alpha \neq \beta$ ). Note that a natural embedding of $S$ in $\Pi_{\alpha \in \Omega} S_{a}$ is given by $\Lambda: s \rightarrow \hat{s}$ where 


$$
\hat{s}(\alpha)= \begin{cases}s & \text { if } s \in S_{\alpha} \\ 0 & \text { otherwise. }\end{cases}
$$

When convenient, we shall identify $S$ with its image in $\Pi_{a \in \Omega} S_{\alpha}$.

For each $\alpha \in \Omega$, let $\mathcal{P}_{\alpha}$ be a right quotient filter on $S_{\alpha}$. The next theorem shows that the collection $\left\{\mathscr{P}_{a}\right\}_{\alpha \in \Omega}$ induces a right quotient filter $\mathscr{P}$ on $S$.

Theorem 6.1. For each $a \in \Omega$, let $\mathfrak{P}_{\alpha}^{\prime}$ be a base for the right quotient filter $\mathcal{P}_{\alpha}$ on $S_{\alpha}$. Let $\mathcal{P}^{\prime}=\left\{A_{S} \subseteq S: A \cap S_{\alpha} \in \mathcal{P}_{\alpha}^{\prime}\right.$ for each $\left.\alpha \in \Omega\right\}$. Then $\mathfrak{P}^{\prime}$ is a base for a right quotient filter $\mathcal{P}$ on $S$ which is independent of the bases $\mathcal{P}_{a}^{\prime}$ taken for $\mathcal{P}_{\alpha}, a \in \Omega$. Furthermore, if $\mathcal{P}_{\alpha}^{\prime}=\mathcal{P}_{\alpha}$ for each $a \in \Omega$ then $\mathcal{P}^{\prime}$ is the right quotient filter $\mathcal{P}$.

Proof. Let $A, B \in \mathcal{P}^{\prime}$. Let $A_{\alpha}=A \cap S_{\alpha}$ and let $B_{\alpha}=B \cap S_{\alpha}$ for each $\alpha \in \Omega$. Let $f \in \operatorname{Hom}_{S}(A, S)$ and let $f_{\alpha}=f \mid A_{\alpha}$. We claim that $f_{\alpha} \in \operatorname{Hom}_{S_{\alpha}}\left(A_{\alpha}, S_{\alpha}\right)$. It is sufficient to show that $f_{a}\left(A_{\alpha}\right) \subseteq S_{\alpha}$. Suppose $f_{\alpha}(s)=t \in S_{\beta}$ where $\beta \neq \alpha$. Then $f_{\alpha}(s) S=t S=0$. Since $S$ has no nonzero null right ideals it follows that $t=0$. Since $A_{\alpha}, B_{\alpha} \in \mathcal{P}_{\alpha}^{\prime}$ and since $\mathcal{P}_{\alpha}^{\prime}$ is a base for $\mathcal{P}_{\text {on }} S_{\alpha}$, there exists $C_{\alpha} \in \mathcal{P}_{\alpha}^{\prime}$ such that $C_{\alpha} \subseteq f_{\alpha}^{-1}\left(B_{\alpha}\right)$. Let $C=\bigcup_{\alpha} C_{\alpha}$. Then $C \in \mathcal{P}^{\prime}$ and clearly $C \subseteq f^{-1}(B)$. Therefore, $\mathcal{P}^{\prime}$ is a base for a right quotient filter $\mathcal{P}$ on $S$. Now suppose that $\mathcal{P}_{\alpha}^{\prime \prime}$ is also a base for $\mathcal{P}_{a}, \alpha \in \Omega$ and let $\mathscr{P}^{\prime \prime}=\left\{A_{S} \subseteq S: A \cap S_{\alpha} \in \mathcal{P}_{a}^{\prime \prime}\right.$ for each $\left.\alpha \in \Omega\right\}$. We claim that each element $A$ of $\mathcal{P}^{\prime}$ contains an element of $\mathcal{P}^{\prime \prime}$. If $A_{a}=A \cap S_{\alpha}$ then $A_{\alpha} \epsilon$ $\mathcal{P}_{\alpha}^{\prime} \subseteq \mathcal{P}_{\alpha}$ and since $\mathcal{P}_{\alpha}^{\prime \prime}$ is also a base for $\mathcal{P}_{\alpha}$ there exists $A_{\alpha}^{\prime} \in \mathcal{P}_{\alpha}^{\prime \prime}$ such that $A_{a}^{\prime} \subseteq A_{a}$. Let $A^{\prime}=\bigcup_{a \in \Omega} A_{a}^{\prime}$. Then $A^{\prime} \in \mathcal{P}^{\prime \prime}$ and we have $A^{\prime} \subseteq A$. A symmetrical argument shows that each element of $\mathcal{P}^{\prime \prime}$ contains an element of $\mathcal{P}^{\prime}$. Thus it follows that $\mathcal{P}^{\prime}$ and $\mathcal{P}^{\prime \prime}$ are both bases for the same R.Q.F. $\mathcal{P}$ on $S$. Finally, suppose that each $\mathcal{P}_{\alpha}$ is an R.Q.F. on $S_{a}$ and let $A, B \in \mathcal{P}^{\prime}$ and $f \in \operatorname{Hom}_{S}(A, S)$. Then

$$
f_{a}=f \mid A \cap S_{a} \in \operatorname{Hom}_{S_{a}}\left(A \cap S_{\alpha}, S_{a}\right)
$$

and

$$
f^{-1}(B)=f^{-1}\left[\bigcup_{a \in \Omega}\left(B \cap S_{a}\right)\right]=\bigcup_{a \in \mathbf{\Omega}}\left[f^{-1}\left(B \cap S_{\alpha}\right)\right]=\bigcup_{a \in \mathbf{\Omega}}\left[f_{a}^{-1}\left(B \cap S_{\alpha}\right)\right] \in \mathcal{P}^{\prime} .
$$

Therefore, in this case $\mathcal{P}^{\prime}$ is an R.Q.F. on $S$ and the theorem follows.

The filter $\mathcal{P}$ on $S$ given by the above theorem will be called the right quo. tient filter induced on $S$ by $\left\{\mathcal{P}_{a}\right\}_{a \in \Omega}$. For the remainder of this section, $\mathcal{P}$ will always denote this induced filter, unless a specific statement to the contrary is made. If each $\mathcal{P}_{\alpha}$ is a standard filter on $S_{\alpha}$ of a given type, the next result shows that $\mathcal{P}$ is the standard filter on $S$ of that type. 
Lemma 6.2. If $\mathscr{P}_{\alpha}$ is $\mathscr{P}_{D}\left(S_{\alpha}\right), \mathscr{P}_{I}\left(S_{\alpha}\right)$, or $\mathcal{P}_{B}\left(S_{\alpha}\right)$ respectively for each $a \in \Omega$ then $\mathcal{P}$ is $\mathcal{P}_{D}(S), \mathcal{P}_{I}(S)$, or $\mathscr{P}_{B}(S)$ in the same order.

Proof. Let $A \in \mathcal{P}_{D}(S)$ and let $A_{\alpha}=A \cap S_{\alpha}$ for each $\alpha \in \Omega$. Let $0 \neq m, n \in S_{\alpha} \subseteq$ $S$. Then there exists $s \in S^{1}$ such that $m s \neq 0$ and $n s \in A$. However, $m \in S_{\alpha}$ and $m s \neq 0$. Hence $s \in S_{\alpha}^{1}$ and it follows that $m s \neq 0$ and $n s \in A \cap S_{\alpha}=A_{\alpha}$. So, we have $A_{\alpha} \in \mathcal{P}_{D}\left(S_{\alpha}\right)$ for each $a$ and it follows that $A \in \mathcal{P}$. Now suppose that $A \in \mathcal{P}$. Then $A_{\alpha}=A \cap S_{\alpha} \in \mathcal{P}_{\alpha}=\mathscr{P}_{D}\left(S_{\alpha}\right)$ for each $\alpha \in \Omega$. Let $0 \neq m, n \in S$, say $m \in S_{\alpha}$ and $n \in S_{\beta}$. If $\alpha=\beta$ then there exists $s \in S_{\alpha}^{1}$ such that $m s \neq 0$ and $n s \in A_{\alpha} \subseteq A$. Suppose $\alpha \neq$ $\beta$. Since $S$ has no nonzero null right ideals there exists $s \in S$ such that $m s \neq 0$. Thus $s \in S_{\alpha}$ and we have $n s=0 \in A$. Hence in either case there exists $s \in S^{1}$ such that $m s \neq 0$ and $n s \in A$. Therefore $A \in \mathcal{P}_{D}(s)$ and it follows that $\mathcal{P}=\mathcal{P}_{D}(S)$. The proof for the remaining cases follows in a similar way from the corresponding definitions and will be omitted.

Returning now to right quotient filters in general, let us see how the $\mathcal{P}_{\text {-tor- }}$ sion congruence on $S$ is determined entirely from the individual $\mathcal{P}_{a}$-torsion congruences on the $S_{a}$ 's.

Proposition 6.3. $\psi_{\boldsymbol{\rho}}(S)=\bigcup_{\alpha} \psi_{\mathcal{P}}\left(s_{\alpha}\right) \cup\left\{(a, b):(a, 0) \in \psi_{\mathcal{P}_{\alpha}}\left(s_{\alpha}\right)\right.$ and $(0, b) \epsilon$ $\psi_{\mathcal{P}}\left(S_{\beta}\right)$ for some $\left.a, \beta \in \Omega\right\}$.

Proof. Let $(a, b) \in \psi_{\mathcal{P}}(S)$. Then there exists $E \in \mathcal{P}$ such that $a s=b s$ for all $s \in E$. If $a$ and $b$ are both in the same $S_{\alpha}$ then $a s=b s$ for all $s \in E \cap S_{\alpha} \in \mathcal{P}_{\alpha}$. Hence $(a, b) \in \psi_{9}\left(S_{\alpha}\right)$. Suppose that $a \in S_{a}$ and $b \in S_{\beta}$ where $\alpha \neq \beta$. Then as = $b s=0$ for all $s \in E \cap S_{\alpha}$ and $b s=a s=0$ for all $s \in E \cap S_{\beta}$. Therefore, in this case $(a, 0) \in \psi_{\rho_{\alpha}}\left(S_{\alpha}\right)$ and $(0, b) \in \psi_{\rho_{\alpha}}\left(S_{\beta}\right)$. Now, if $(a, b) \in \psi_{\rho_{a}}\left(S_{\alpha}\right)$ for some $\alpha \epsilon$ $\Omega$ then there exists $A_{\alpha} \in \mathcal{P}_{a}$ such that $a A_{\alpha}=b A_{\alpha}$. Let $A=A_{\alpha} \cup\left(\cup_{\beta \neq a} S_{\beta}\right)$. Then $A \in \mathcal{P}$ and $a A=b A$. Thus we have $(a, b) \in \psi_{\rho}(S)$ and it follows that $\psi_{\mathcal{P}_{\alpha}}\left(S_{\alpha}\right) \subseteq$ $\psi_{\rho}(S)$. The remaining inclusion is immediate from this and the transitivity of $\psi_{\rho}(S)$.

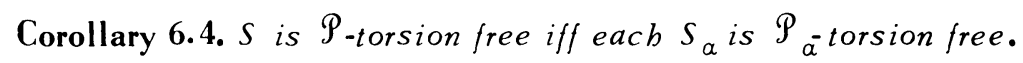

The next theorem shows that the semigroup of right quotients of $S$ with respect to $\mathcal{P}$ is determined by the semigroups $\left\{Q_{\Phi_{\alpha}}\left(S_{\alpha}\right)\right\}_{\alpha \in \Omega}$.

Theorem 6.5. There exists an isomorpbism $\Phi$ from $\Pi_{a \in \mathbf{\Omega}} Q_{\Phi_{\alpha}}\left(S_{\alpha}\right)$ onto $Q_{\mathcal{P}}(S)$, under which $S$ is invariant when $\psi_{\mathcal{\rho}}(S)=\imath$.

Proof. Let $\left(\bar{f}_{\alpha}\right)_{a \in \mathbf{\Omega}} \in \prod_{a} Q_{\mathcal{P}_{\alpha}}\left(S_{\alpha}\right)$ which we abbreviate as $\left(\bar{f}_{\alpha}\right)$. Let $D_{f}=$ $\cup_{a} D_{f_{a}} \in \mathcal{P}$ and define $f: D_{f} \rightarrow S$ by $f(x)=f_{a}(x)$ where $x \in D_{f_{a}}$. Then $f \epsilon$ $\operatorname{Hom}_{S}\left(D_{f}, S\right)$. Define $\Phi: \Pi_{a} Q_{\Phi_{\alpha}}(s) \rightarrow Q_{\mathcal{P}}(s)$ by $\Phi\left(\left(\bar{f}_{a}\right)\right)=\bar{f}$. It is easily seen that $\Phi$ is the desired isomorphism. 
The invariance of $S$ under $\Phi$ when $\psi_{\rho}(S)=\iota$ is shown in the diagram below for an arbitrary element $s$ of $S$.

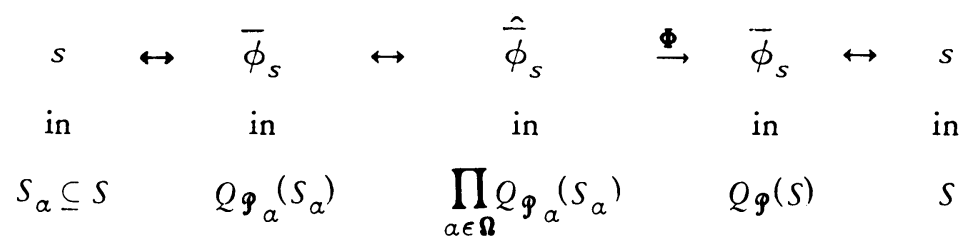

For each $\alpha \in \Omega$, let $T_{\alpha}$ be a semigroup such that $S_{\alpha} \subseteq T_{\alpha}$. A semigroup $T$ containing $S$ as a subsemigroup is called a subdirect product of $\left\{T_{\alpha}\right\}_{\alpha \in \Omega}$ over $S$ if there exists an isomorphism $\theta$ from $T$ into $\Pi_{a \in \mathbf{\Omega}} T_{a}$ such that

(a) $\theta \mid S=\iota_{S}$ (under the identification given at the beginning of this section), and

(b) $\pi_{\alpha} \theta(T)=T_{\alpha}$ for each $\alpha \in \Omega$, where $\pi_{\alpha}$ is the projection mapping of $\Pi_{\alpha \in \Omega} T_{a}$ onto $T_{\alpha}$.

We will now show that subdirect products over $S$ of $\mathcal{P}_{\alpha^{-} \text {torsion free }} \mathcal{P}_{\alpha^{\text {-quo- }}}$ tient semigroups over $S_{\alpha}, \alpha \in \Omega$, determine up to isomorphism all $\mathcal{P}_{\text {-torsion free }}$ $\mathcal{P}$-quotient semigroups over $S$. The following lemma which can easily be shown will be useful in the proof. It is valid for any semigroup $S$ with zero and any right quotient filter $\mathcal{P}$ on $S_{S}$.

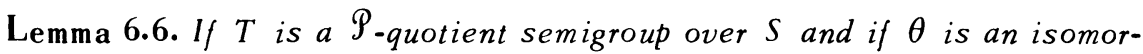
phism from $T$ onto a semigroup $T^{\prime}$ containing $S$ sucb that $\theta \mid S={ }^{\prime} s$ then $T^{\prime}$ is a

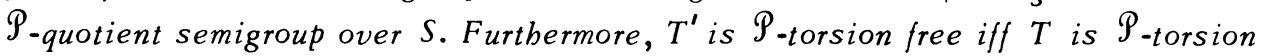
free.

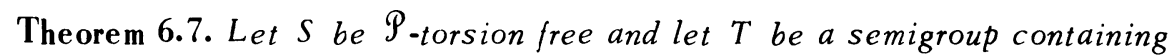

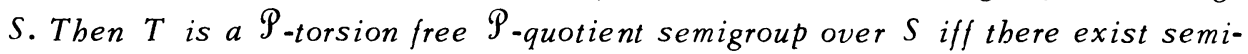
groups $T_{\alpha}$, a $\in \Omega$, such that each $T_{\alpha}$ is a $\mathcal{P}_{\alpha}$-torsion free $\mathcal{P}_{\alpha}$-quotient semigroup over $S_{a}$ and $T$ is a subdirect product over $S$ of $\left\{T_{a}\right\}_{a \in \Omega}$.

Proof. $(\Rightarrow)$ By Corollary 5.8 there exists a semigroup $T^{\prime}$ isomorphic to $T$ such that $S \subseteq T^{\prime} \subseteq Q_{\rho}(S)$ and $S$ is invariant under the given isomorphism. Let $T^{\prime \prime}=\Phi^{-1}\left(T^{\prime}\right)$ where $\Phi$ is given in Theorem 6.5. Hence we have $S \subseteq T^{\prime \prime} \subseteq$ $\mathrm{Il}_{\alpha \in \boldsymbol{\Omega}} Q_{\boldsymbol{\Phi}_{\alpha}}\left(S_{\alpha}\right)$. For each $\alpha \in \Omega$, let $T_{\alpha}=\pi_{\alpha}\left(T^{\prime \prime}\right)$ where $\pi_{\alpha}$ is the projection of II ${ }_{\alpha \in \Omega} Q_{\mathcal{P}_{\alpha}}\left(S_{\alpha}\right)$ onto $Q_{\boldsymbol{P}_{\alpha}}\left(S_{\alpha}\right)$. Thus we have $S_{\alpha} \subseteq T_{\alpha} \subseteq Q_{\boldsymbol{\Phi}_{\alpha}}\left(S_{\alpha}\right)$ for each $\alpha \in \Omega$. Since $Q_{\varphi_{\alpha}}\left(S_{\alpha}\right)$ is a $\mathcal{P}_{\alpha}$-torsion free $\mathcal{P}_{\alpha}$-quotient semigroup over $S_{\alpha}$, it clearly follows that each $T_{\alpha}$ is also a $\mathcal{P}_{\alpha}$-torsion free $\mathcal{P}_{\alpha}$-quotient semigroup over $S_{\alpha}$. Furthermore, it is straightforward to see that $T$ is a subdirect product over $S$ of the $\left\{T_{\alpha}\right\}_{\alpha \in \mathbf{\Omega}}$.

$(\Leftarrow)$ By Lemma 6.6, we may assume that $S \subseteq T \subseteq \Pi_{\alpha \in \Omega} T_{\alpha}$ such that $\pi_{\alpha}(T)=$ $T_{\alpha}$.

Recall that in this context we are regarding $S$ as a subset of $\Pi_{\alpha \in \boldsymbol{\Omega}} S_{\alpha} \subseteq$ 
$\Pi_{\alpha \in \boldsymbol{\Omega}} T_{\alpha}$ under the identification $s \leftrightarrow \hat{s}$. Let $t \in T$ be arbitrary. Since $T_{\alpha}$ is a $\mathcal{P}_{\alpha}$ quotient semigroup over $S_{\alpha}$ for each $\alpha$, it follows that $t(\alpha)^{-1} S_{\alpha} \in \mathcal{P}_{\alpha}$. Let $E=$ $\bigcup_{\alpha \in \mathbf{\Omega}} t(\alpha)^{-1} S_{\alpha}$. Then $E \in \mathcal{P}$ and for each $s \in E \cap S_{\alpha}, \alpha \in \Omega$, we have

$$
t s(\beta)= \begin{cases}0 & \text { if } \beta \neq \alpha, \\ t(\alpha)_{s} \in s_{\alpha} & \text { if } \beta=\alpha .\end{cases}
$$

Hence it follows that $s \in t^{-1} S$. Thus $E \subseteq t^{-1} S$ and $t^{-1} S \in \mathcal{P}$ and we see that $T$ is a $\mathcal{P}$-quotient semigroup over $S$. In order to show that $T$ is $\mathcal{P}_{\text {-torsion free, sup- }}$ pose that $t, t^{\prime} \in T$ and $E \in \mathcal{P}$ such that $t E \div t^{\prime} E$. We claim that $t(\alpha)=t^{\prime}(\alpha)$ for each $\alpha \in \Omega$. Let $E_{\alpha}=E \cap S_{\alpha} \in \mathcal{P}_{\alpha}$. Then for each $s \in E_{\alpha}$, we have $t(\alpha) s=$ $t(\alpha) \hat{s}(\alpha)=(t \hat{s})(\alpha)=\left(t^{\prime} s\right)(\alpha)=t^{\prime}(\alpha) \hat{s}(\alpha)=t^{\prime}(\alpha) s$. Hence $\left(t(\alpha), t^{\prime}(\alpha)\right) \in \psi_{\rho}\left(s_{\alpha}\right)=$ $\iota$ and the claim follows. Therefore, $t=t^{\prime}$ and it follows that $T$ is $\mathfrak{P}_{\text {-torsion free. }}$

7. Primitive dependent semigroups. Let $S$ be a semigroup and let $E=E(S)$ be the set of idempotents in $S$. A partial ordering $\leq$, called the natural partial ordering on $E$, is defined by $e \leq f$ iff $e=f e=e f$. An element $0 \neq e \in E$ is said to be primitive if $0 \leq f \leq e, f \in E$, implies that $f=0$ or $f=e$. The set of primitive idempotents of $S$ will be denoted by $E^{\prime}=E^{\prime}(S)$. A regular semigroup $S$ is said to be primitive regular if $E^{\prime}=E \backslash\{0\}$. An important subclass of the class of primitive regular semigroups is the collection of all completely 0 -simple semigroups. These semigroups have been characterized as regular Rees matrix semigroups over groups with 0 as shown in Chapter III of Clifford and Preston [4].

A nonzero regular semigroup $S$ will be called primitive dependent if for each $0 \neq e \in E=E(S)$ there exists $e^{\prime} \in E^{\prime}=E^{\prime}(S)$ such that $e^{\prime} \leq e$. This class of semigroups contains the primitive regular semigroups, all finite regular semigroups, and, as will be shown, all completely semisimple semigroups with principal series.

In this section we shall develop several theorems involving the singular congruence on a completely 0 -simple semigroup. In addition, a characterization of the completely 0 -simple $\mathcal{P}_{l}$-torsion free semigroups will be given. These facts along with the general theorems given in $\$ 6$ will lead to a complete characterization of all primitive dependent $\mathscr{P}_{I}$-torsion free semigroups as regular subdirect products of column monomial matrix semigroups over groups.

We begin with a few definitions and lemmas which can be stated for $S$-sets in general. A nonzero sub $S$-set $N_{S}$ of an $S$-set $M_{S}$ is said to be 0 -minimal if $N_{S}$ contains no proper nonzero sub $S$-sets. Such $S$-sets are characterized as follows.

Lemma 7.1. Let $0 \neq N_{S} \subseteq M_{S}$. Then $N_{S}$ is 0 -minimal iff for each $0 \neq x, y \in$ $N$, there exists $s \in S^{1}$ such that $y=x s\left(i . e\right.$. , iff $N=x S^{1}$ for eacb $\left.0 \neq x \in N\right)$.

Proof. $(\Rightarrow)$ Suppose $N$ is 0 -minimal. Let $0 \neq x \in N$. Then $0 \subsetneq x S^{1} \subseteq N$. Hence $x S^{1}=N$ since $N$ is 0 -minimal. 
$(\Leftarrow)$ Let $0 \subsetneq N^{\prime} \subseteq N$ and let $n \in N$ and $0 \neq n^{\prime} \in N^{\prime}$. Then there exists $s \in$ $S^{1}$ such that $n=n^{\prime} s \in N^{\prime}$. Thus $N^{\prime}=N$ and the result follows.

The right socle $\Sigma=\Sigma_{r}(M)$ of an $S$-set $M$ is the union of $\{0\}$ and all 0 -minimal sub $S$-sets of $M_{S}$. The left socle $\Sigma_{l}(M)$ is defined dually for left $S$-sets.

Lemma 7.2. $\Sigma_{r}(M) \subseteq N$ for every $N \in \mathscr{P}_{I}\left(M_{S}\right)$.

Proof. Let $0 \neq m \in \Sigma_{r}(M)$. Then $m S^{1} \cap N \neq 0$ and since $m S^{1}$ is 0 -minimal it follows that $m \in m S^{1} \subseteq N$. Therefore, $\Sigma_{r}(M) \subseteq N$.

Lem ma 7.3. $M=\Sigma_{r}(M)$ iff $\mathscr{P}_{I}\left(M_{S}\right)=\{M\}$.

Proof. ( $\Rightarrow$ ) This is immediate from Lemma 7.2.

$(\Leftarrow)$ Let $0 \neq m \in M$. We claim that $m S^{1}$ is 0 -minimal. Let $0 \neq m s_{1}, m s_{2} \epsilon$ $m S^{1}$. As in the proof of Proposition 4.7, there exists a sub $S$-set $N$ of $M$ such that $m s_{1} S^{1} \cap N=0$ and $m s_{1} S \cup N \in \mathscr{P}_{I}\left(M_{S}\right)$. Hence, since $\mathscr{P}_{I}\left(M_{S}\right)=\{M\}$, we have $m s_{1} s^{1} \cup N=M$. If $m \in N$ then $m s_{1} \in m s_{1} S^{1} \cap N=\{0\}$ which is a contradiction. Thus, $m \in m s_{1} s^{1}$ and it follows that $m s_{2} \in m s_{1} s^{1}$. Therefore, $m S^{1}$ is 0 -minimal by Lemma 7.1 and $m \in m S^{1} \subseteq \Sigma_{r}(M)$.

Note that if $\Sigma=\Sigma_{r}(M) \in \mathcal{P}_{I}^{r}\left(M_{S}\right)$ then, by Lemma 5.2, $Q_{l}\left(M_{S}\right) \approx \operatorname{Hom}_{S}(\Sigma, \Sigma)$ under the map $\Phi(f)=\bar{f}$ where $f \in \operatorname{Hom}_{S}(\Sigma, \Sigma)$. Hereafter, we shall regard this isomorphism as an identity.

The theorem which follows determines all regular semigroups $S$ for which $\Sigma_{r}(S)=S$. Parts (a), (b), (c), and (d) of the theorem are taken from Theorem 6.39 of Clifford and Preston [5]. The equivalence of $(c)$ and $(e)$ is given by Lemma 7.3 .

Theorem 7.4. The following are equivalent where $S=S^{0}$ is a semigroup.

(a) $S$ is a 0-direct union of completely 0-simple semigroups.

(b) $S$ is a union of 0-minimal right ideals of the form eS where $e^{2}=e \in S$.

(c) $S$ is regular and $\Sigma_{r}(S)=S$.

(d) $S$ is primitive regular.

(e) $S$ is regular and $\mathcal{P}_{I}(S)=\{S\}$.

From part (e) of the above theorem we note that the terms "right reductive" and "P $P_{I}$-torsion free" are equivalent on a primitive regular semigroup. The next theorem, which describes the socle of any regular semigroup, is essentially a restatement of Theorem 7.59 in Clifford and Preston [5].

Theorem 7.5. Let $S$ be a regular semigroup with 0 . Then $\Sigma_{r}(S)=\Sigma_{l}(S)$ and the socle $\Sigma\left(=\Sigma_{r}(S)=\Sigma_{l}(S)\right)$, if nonzero, is the largest primitive regular ideal of $S$ and it contains all the primitive idempotents of $S$.

Note that if $E^{\prime}=E^{\prime}(S) \neq \square$ then $\Sigma(S)=S E^{\prime} S=E^{\prime} S=S E^{\prime}$ for any regular semigroup $S$. 
The preceding two theorems indicate that completely 0 -simple semigroups are some of the basic "building blocks" for all regular semigroups with nonzero socle. Furthermore, the well-known Rees theorem given on p. 94 of [4] describes each completely 0 -simple semigroup as a regular Rees matrix semigroup $\mathfrak{M}^{0}(G, I, J ; P)$ over a group with 0 . For this reason we begin out study of the singular congruence and the essential semigroup of quotients on a regular semigroup by considering first the case where $S$ is a regular Rees matrix semigroup.

Let $S=M^{0}(G, I, J ; P)$ be the regular Rees matrix semigroup over the group $G$ with zero, having indices $I$ and $J$ and $J \times I$ sandwich matrix $P$. The nonzero elements of $S$ will be written in the form $A=(a)_{i j}$ where $a \in G$ is the single nonzero entry in the $I \times J$ matrix $A$ which appears in the $i$ th row and $j$ th column. The element in the $(j, i)$ position of $P$ will be denoted by $p_{j i}$. Row $j_{1}$ of $P$ is said to be left proportional to row $j_{2}$ of $P$ if there exists $c \in G$ such that $p_{j_{1} i}=$ $c p_{j_{2} i}$ for all $i \in I$. Dually, column $i_{1}$ is right proportional to column $i_{2}$ if there exists $c \in G$ such that $p_{j i_{1}}=p_{j i_{2}} c$ for all $j \in J$. The next theorem determines the singular congruence on $S$.

Theorem 7.6. For $S=\mathbb{M}^{0}(G, I, J ; P), \psi_{I}(S)=\left\{\left[(a)_{i j_{1}},(b)_{i j_{2}}\right]:\right.$ row $j_{1}$ is left proportional to row $j_{2}$, say $p_{j_{1} i}=c p_{j_{2} i}$ for each $i \in I$, and $a, b \in G$ such that $\left.a^{-1} b=c\right\} \cup\{(0,0)\}$.

Proof. Since $S$ is regular, $\psi_{I}(S)$ is 0 -restricted by Lemma 4.6. Let $\left[(a)_{i_{1} j_{1}}\right.$, $\left.(b)_{i_{2} j_{2}}\right] \in \psi_{I}(S)$. Since $\mathcal{P}_{I}(S)=\{S\}$, we have $(a)_{i_{1} j_{1}}(x)_{i j}=(b)_{i_{2} j_{2}}(x)_{i j}$ for each $(x)_{i j} \in S$. Hence $\left(a p_{j_{1} i} x\right)_{i_{1} j}=\left(b p_{j_{2} i} x\right)_{i_{2 j}}$ for all $(x)_{i j} \in S$. Thus, it follows that $i_{1}=i_{2}$ and $a p_{j_{1} i}=b p_{j_{2} i}$ for each $i \in I$. Therefore, $p_{j_{1} i}=a^{-1} b p_{j_{2} i}=c p_{j_{2} i}$ for each $i \in I$, where $c=a^{-1} b$, and we see that $\left[(a)_{i_{1} j_{1}},(b)_{i_{1} j_{2}}\right]$ is a member of the right side. The opposite inclusion is immediate from retracing the above steps.

Corollary 7.7. If $P$ consists entirely of 0 's and 1's then $\psi_{I}(S)=\left\{\left[(x)_{i j_{1}}\right.\right.$, $(x)_{i j_{2}}$ ]: rows $j_{1}$ and $j_{2}$ of $P$ are equal $\} \cup\{(0,0)\}$.

Corollary 7.8. $\psi_{I}(S)=\iota$ iff no two rows of $P$ are left proportional.

Corollary 7.9. If $S$ is a primitive inverse semigroup then $\psi_{I}(S)=\iota$.

Proof By Theorem 7.4, $S$ is a 0 -direct union of $\left\{S_{\alpha}\right\}_{a \in \mathbf{\Omega}}$ where each $S_{\alpha}$ is completely 0-simple. Since $S_{\alpha} \subseteq S$, it follows that $S_{\alpha}$ is a Brandt semigroup for each $\alpha \in \Omega$. Hence by Theorem 3.9 of Clifford and Preston [4], $S_{\alpha}$ is isomorphic to a regular Rees matrix semigroup $\mathbb{M}^{0}\left(G_{a}, I_{a}, I_{a} ; \triangle_{a}\right)$ over a group $G_{a}$ with 0 and with the $I_{\alpha} \times I_{\alpha}$ identity matrix $\triangle_{\alpha}$ as sandwich matrix. Thus, from Corollary 7.8 we see that $\psi_{I}\left(S_{\alpha}\right)=\imath$ for each $\alpha \in \Omega$. Therefore, the result follows by Lemma 6.2 and Corollary 6.4. 
Gordon L. Bailes [2] has defined a right inverse semigroup as a regular semigroup $S$ in which each element has a unique left unit (i.e., $|x V(x)|=1$ where $V(x)$ is the set of inverses of $x$ in $S)$. A left inverse semigroup is defined dually. Corollary 4 of [2] shows that $S$ is an inverse semigroup iff $S$ is both a left and a right inverse semigroup.

Theorem 7.10. Let $S$ be a completely 0 -simple semigroup. Then the following are equivalent.

(a) $S$ is right inverse.

(b) $S$ is isomorphic to a regular Rees matrix semigroup $\mathbb{M}^{0}(G, I, J ; P)$ where $P$ consists entirely of 0 's and 1's and each column contains exactly one nonzero entry.

(c) $\psi_{I}(S)=\imath$ and $S$ is ortbodox.

Proof. (a) $\Leftrightarrow$ (b) is essentially a restatement of Theorem 44 in [2] and (b) $\Leftrightarrow$ (c) is immediate from Corollary 7.8 and Theorem 6 of [7].

Corollary 7.11. A primitive regular semigroup is a right inverse semigroup iff $\psi_{I}(S)=\iota$ and $S$ is ortbodox.

Proof. By Theorem 7.4, $S$ is a 0 -direct union of completely 0 -simple semigroups $\left\{S_{a}\right\}_{a \in \Omega}$. Clearly, by the definition $S$ is a right inverse semigroup iff each $S_{\alpha}$ is a right inverse semigroup. By the theorem this is true iff each $S_{\alpha}$ is orthodox and $\psi_{I}\left(S_{\alpha}\right)=\imath$. Finally, by Corollary 6.4, this follows iff $S$ is orthodox and $\psi_{I}(S)=\iota$.

Corollary 7.12. An orthodox primitive regular semigroup is inverse iff $\psi_{I}(S)=$ $\iota$ and ${ }_{I} \psi(S)=\iota$, where ${ }_{I} \psi(S)$ is the left-right dual of $\psi_{I}(S)$.

Let $S=\mathbb{M}^{0}(G, I, J ; P)$ be a regular Rees matrix semigroup and let $\mathcal{C}^{0}(G, I)$ denote the semigroup of all $I \times I$ column monomial matrices over $G^{0}$. If $A \epsilon$ $\mathcal{C}^{0}(G, I)$ then $A(i, j)$ will denote the element in the $(i, j)$ position of $A$. Multiplication in $S$ will be indicated by juxtaposition whereas ordinary multiplication between matrices as defined on p. 87 of Clifford and Preston [4] will be denoted by "०". Hence, if $A, B \in S$ then $A B=A \circ P \circ B$. Since $S=\Sigma(S)$ in this case, $\mathcal{P}_{I}(S)=\{S\}$ and we have $Q_{I}(S)=\operatorname{Hom}_{S}(S, S)$ which is precisely the semigroup $\Lambda(S)$ of all left translations of $S$. This has been characterized by Petrich [13] as a wreath product over a group. Another characterization of $\Lambda(S)$ is also given in Proposition 4.5 .6 of [13] which is restated below without proof.

Theorem 7.13. Let $S=\mathbb{M}^{0}(G, l, J ; P)$ be a regular Rees matrix semigroup. Then there exists an isomorphism $\Phi: \operatorname{Hom}_{S}(S, S) \stackrel{\text { onto }}{\longrightarrow} \mathcal{C}^{0}(G, I)$, say $\Phi(f)=C_{f}$, such that $f(X)=C, \circ X$ for each $f \in \operatorname{Hom}_{S}(S, S)$ and for each $X \in S$. 
Let $\phi$ denote the representation of $S$ in $Q_{I}(S)=\operatorname{Hom}_{S}(S, S)$ given in $\$ 5$. Then $\Phi \phi$ is a representation of $S$ in $C^{0}(G, I)$. The image of this representation is given by the following proposition.

Proposition 7.14. Let $\Phi \phi$ be the representation of $S$ in $\mathcal{C}^{0}(G, I)$ given above. For each $0 \neq X=(x)_{i j} \in S$ let $C_{X}$ denote the element of $\mathcal{C}^{0}(G, I)$ given by

$$
C_{X}=\left(i^{\prime}, k\right)= \begin{cases}0 & \text { if } i \neq i^{\prime} \in I, k \in I, \\ x p_{j k} & \text { if } i=i^{\prime}, k \in I .\end{cases}
$$

Then $\Phi \phi(X)=C_{X}$.

Proof. Let $f \in Q_{I}(S)$ such that $\Phi(f)=C_{X}$. We claim that $f=\phi_{X}$. Let $Y=$ $(y)_{i{ }^{\prime}{ }^{\prime}}$ be an arbitrary element of $S$. Then

$$
\begin{aligned}
f(Y) & =C_{f} \circ Y=C_{X} \circ Y=\left(\begin{array}{lll}
0 & & 0 \\
& x p_{j i^{\prime}} & \\
0 & & 0
\end{array}\right) \circ\left(\begin{array}{cc}
0 & 0 \\
0 \cdots 0 & y 0 \cdots 0 \\
0 & 0
\end{array}\right) \\
& =\left(x p_{j i}, y\right)_{i j^{\prime}}=X Y=\phi_{X}(Y) .
\end{aligned}
$$

Therefore, $f=\phi_{X}$ and the proposition follows.

Note that if $S=\pi^{0}(G, I, I ; \Delta)$ where $\Delta$ is the $I \times I$ identity matrix then $\Phi \phi(X)=X$ for each $X \in S$, i.e., the representation of $S$ is actually the identity map.

The above proposition leads us to the following definition.

Let $G$ be a group, $I$ and $J$ be index sets, and let $P$ be a regular $J \times I$ matrix over $G^{0}$ such that no two rows of $P$ are left proportional. Let $\mathcal{H}^{0}=$ $\mathcal{H}^{0}(G, I, J ; P)$ denote the subset of all elements " $A$ " in $\mathcal{C}^{0}(G, I)$ such that for some $i \in I, j \in J$, and $g \in G^{0}$,

$$
A\left(i^{\prime}, k\right)= \begin{cases}0 & \text { if } i^{\prime} \neq i, k \in I, \\ g p_{j k} & \text { if } i^{\prime}=i, k \in I .\end{cases}
$$

If $S$ is the semigroup $\mathbb{M}^{0}(G, I, J ; P)$ then $S$ is $P_{I^{-t o r s i o n}}$ free since no two rows of $P$ are left proportional. Hence $\mathcal{H}^{0}$ is the isomorphic image of $S$ under the map $\Phi \phi$ into the semigroup $C^{0}(G, I)$. Thus since $\phi(S)$ is a left ideal of $Q_{I}(S)=\operatorname{Hom}_{S}(S, S)$, we see that $\mathcal{H}^{0}$ is a left ideal of $\mathcal{C}^{0}(G, I)$. Furthermore, a trace of the isomorphisms involved shows that $\mathcal{C}^{0}(G, I)$ is isomorphic over $\mathcal{H}^{0}$ to $Q_{I}\left(\mathcal{H}^{0}\right)$. The semigroup $\mathcal{H}^{0}$ will be called an $\mathcal{H}$-semigroup. The next theorem follows from the discussion above and $\S 5$.

Theorem 7.15. Let $\mathcal{H}^{0}=\mathfrak{H}^{0}(G, I, J ; P)$ be an arbitrary $\mathcal{H}$-semigroup. Then 
(a) A semigroup $S$ is completely 0 -simple right reductive iff $S$ is isomorphic to some $\mathcal{H}$-semigroup.

(b) $\mathcal{H}^{0}$ is a left ideal of $\mathfrak{C}^{0}(G, I)$.

(c) $\mathcal{C}^{0}(G, I)$ is isomorphic over $\mathfrak{H}^{0}$ to $Q_{I}\left(\mathcal{H}^{0}\right)$.

(d) $\mathfrak{C}^{0}(G, I)$ is the injective bull of $\mathcal{H}^{0}$.

(e) $\mathcal{C}^{0}(G, I)$ is self-injective.

These results can also be applied to primitive regular semigroups as stated below. The proofs are immediate from the general theory developed in $\$ 6$ since each primitive regular semigroup is a 0 -direct union of completely 0 -simple semigroups.

Theorem 7.16. Let $S$ be a primitive regular semigroup. Then

(a) $S$ is primitive regular right reductive iff $S$ is isomorpbic to a 0 -direct union of $\mathcal{H}$-semigroups.

(b) $Q_{I}(S)=\operatorname{Hom}_{S}(S, S)$ and is isomorphic to a direct product of column monomial matrix semigroups over groups.

We shall now consider primitive dependent semigroups. Certainly any primitive regular semigroup is primitive dependent, as well as any finite regular semigroup. A more general class of primitive dependent semigroups is given in the theorem which follows. The reader is referred to $\$ \$ 2.6$ and 6.6 of Clifford and Preston [4] and [5] for the definitions of the terms used.

Theorem 7.17. Let $S=S^{0}$ be a completely semisimple semigroup with a principal series. Then $S$ is a primitive dependent semigroup.

Proof. Let $S=S_{1} \supset S_{2} \supset \ldots \supset S_{n}=\{0\}$ be a principal series for $S$. We shall proceed by induction on $n$. If $n=2$, then $S=S_{1}$ is completely 0 -simple and the result follows. Suppose the theorem is true for all completely semisimple semigroups having a principal series of length $k$ where $2 \leq k<n, n>2$, and let $S$ have a principal series of length $n$ as above. Then $S_{2}$ has a principal series of length $n-1$. Let $0 \neq e=e^{2} \in S=S_{1}$. If $e \in S_{2}$ then by the induction hypothes is there exists an idempotent $f^{\prime} \in S_{2}$ such that $f^{\prime} \leq e$ and such that $f^{\prime}$ is primitive in $S_{2}$. Since $S_{2}$ is an ideal of $S$, it follows that $f^{\prime}$ is also primitive in $S_{1}$. Hence the result is true in this case. Now suppose $e \in S \backslash S_{2}$. If $e$ is primitive in $S$, we are through. So, suppose $e$ is not primitive in $S$. Then there exists $0 \neq f \in E(S)$ such that $0 \neq f<e$. If $f \in S \backslash S_{2}$ then $0 \neq f<e$ in $S / S_{2}=S_{1} / S_{2}$ which is completely 0 simple. But this is impossible. Hence $f \in S_{2}$ and by the induction hypothes is there exists $f^{\prime}$ primitive in $S_{2}$ (hence in $S$ ) such that $f^{\prime} \leq f$. Therefore $f^{\prime}<e$ and the theorem is proved.

Let $R$ be a subset of a semigroup $S$. The left annihilator of $R$ in $S$ is ${ }_{R} A=$ 
$\{x \in S: x R=0\}$. The right annihilator of $R$ is defined dually and is denoted by $A_{R}$. Several equivalences to the definition of primitive dependent can now be stated.

Theorem 7.18. Let $S$ be a regular semigroup and let $\Sigma$ be the socle of $S$. Then the following are equivalent.

(a) $\Sigma_{\Sigma} A=0$.

(b) $\Sigma \in \mathcal{P}_{I}\left(S_{\Sigma}\right)$.

(c) $\Sigma \in \mathscr{P}_{l}(S)$.

(d) Every nonzero right ideal of $S$ contains a 0 -minimal right ideal of $S$.

(e) $S$ is primitive dependent.

Proof $(\mathrm{a}) \Rightarrow(\mathrm{b})$. Let $0 \neq s \in S$. Since $\Sigma A=0$, there exists $x \in \Sigma$ such that $s x \neq 0$ and since $\Sigma$ is a 2 -sided ideal of $S$, we have that $0 \neq s x \in \Sigma$. Thus it follows that $\Sigma \in \mathcal{P}_{I}\left(S_{\Sigma}\right)$.

(b) $\Rightarrow(c)$. This is immediate from Theorem 3.7 .

$(\mathrm{c}) \Rightarrow(\mathrm{d})$. Let $R$ be a nonzero right ideal of $S$. Since $\Sigma \in \mathcal{P}_{I}(S)$, there exists $0 \neq x \in R \cap \Sigma$, and since $x \in \Sigma, x S^{1}$ is 0 -minimal. Furthermore, $x S^{1} \subseteq R$.

$(\mathrm{d}) \Rightarrow(\mathrm{e})$. Let $0 \neq e \in E$. By the hypothes is eS contains a 0 -minimal right ideal $R$ of $S$. Since $S$ is regular, $R$ is of the form $R=f S$ for some $0 \neq f \in E$, and since $f \in \Sigma$, we have that $f \in E^{\prime}$. Furthermore, $e f=f$. Let $f^{\prime}=f e$. Then $f^{\prime} \in E$ since $f^{\prime 2}=(f e)(f e)=f(e f) e=f f e=f e=f^{\prime}$, and $f^{\prime} f=f e f=f f=f \neq 0$. Hence $f^{\prime} \neq 0$. Furthermore, $f^{\prime}=f e \in f S \subseteq \Sigma$. Thus it follows that $f^{\prime} \in E^{\prime}$. Finally, $f^{\prime} e=$ $f e e=f e=f^{\prime}=f e=e f e=e f^{\prime}$. Therefore, $0 \neq f^{\prime} \leq e$ and $f^{\prime} \in E^{\prime}$ and it follows that $S$ is primitive dependent.

(d) $\Rightarrow(\mathrm{a})$. Let $0 \neq x \in S$ and let $x^{\prime}$ be an inverse of $x$. Then there exists $f \epsilon$ $E^{\prime}$ such that $f \leq x^{\prime} x$. Hence $x^{\prime} x f=f \in E^{\prime} \subseteq \Sigma$. Therefore, $0 \neq x f \in x \Sigma$ and it follows that $\Sigma_{\Sigma} A=0$.

Note that the left-right duals of (a), (b), (c) and (d) respectively are equivalent to (e) also.

The essential semigroup of quotients of a primitive dependent semigroup is given by the following lemma.

Lemma 7.19. Let $S$ be primitive dependent and let $\Sigma$ be its socle. Then $Q_{I}(S)$ is semigroup isomorpbic to $\operatorname{Hom}_{\Sigma}(\Sigma, \Sigma)=Q_{I}(\Sigma)$.

Proof. From a note following Lemma 7.3 and from the above theorem we see that $Q_{I}(S) \approx \operatorname{Hom}_{S}(\Sigma, \Sigma)$. Hence it is sufficient to show that every $\Sigma$-homomorphism on $\Sigma$ is an $S$-homomorphism. Let $f \in \operatorname{Hom}_{\Sigma}(\Sigma, \Sigma), t \in \Sigma$, and $s \in S$. Then for $t^{\prime}$, an inverse of $t$ in $\Sigma$, we have $f(t s)=f\left(t t^{\prime} t s\right)=f(t) t^{\prime} t s=f\left(t t^{\prime} t\right) s=f(t) s$ and the result follows.

Another characterization of the singular congruence can be given for a primitive dependent semigroup. 
Lemma 7.20. If $S$ is primitive dependent then $\psi_{I}(S)=\{(x, y): x e=y e$ for all $\left.e \in E^{\prime}\right\}$.

Proof. By Lemma 7.2 and Theorem 7.18, $\{\Sigma\}$ is a base for $\mathscr{P}_{I}(S)$. Thus by Lemma 4.1, $\psi_{I}(s)=\{(x, y): x s=y s$ for all $s \in \Sigma\}$. Since $E^{\prime} \subseteq \Sigma$, it follows that $\psi_{I}(S)$ is contained in the right side above. Now, let $(x, y)$ be in the right side and let $s \in \Sigma$. Then there exists $0 \neq e=e^{2} \in \Sigma$ such that $s=e s$ and, since $e \in \Sigma$, $e \in E^{\prime}$. Therefore $x s=x e s=y e s=y s$ and $(x, y) \in \psi_{I}(s)$.

Lemma 7.21. Let $S \subseteq T$ be regular semigroups such that $S$ is a left (right, 2-sided) ideal of $T$. Then $\Sigma(S) \subseteq \Sigma(T)$.

Proof. Let $e \in E^{\prime}(S)$. We claim that $e \in E^{\prime}(T)$. Suppose there exists $f^{2}=f \epsilon$ $T$ such that $f \leq e$. Then $f=f e=e f \in S$ in any case above. Hence, since $e$ is primitive in $S$, it follows that $f=0$ or $f=e$. Therefore, $e \in E^{\prime}(T)$ and we see that $\Sigma(S)=E^{\prime}(S) S \subseteq E^{\prime}(T) T=\Sigma(T)$ by the note following Theorem 7.5.

The next theorem shows that certain regular $\mathcal{P}_{I}$-quotient semigroups over a primitive dependent semigroup are also primitive dependent.

Theorem 7.22. Let $S$ be a primitive dependent semigroup and let $T$ be any regular $\mathcal{P}_{I}(S)$-quotient semigroup over $S$ such that $S$ is a left (right, 2-sided) ideal of $T$ and $\psi_{I}\left(T_{S}\right)$ is 0-restricted. Then $T$ is primitive dependent with socle $\Sigma(T)=\Sigma(S) T^{1}$.

Proof. By Lemma 7.21, $\Sigma(S) \subseteq \Sigma(T)$. Hence $\Sigma(S) T^{1} \subseteq \Sigma(T)$. We claim that $S \in \mathscr{P}_{I}\left(T_{S}\right)$. Let $0 \neq t \in T$. Then since $T$ is a $\mathscr{P}_{l}$-quotient semigroup over $S$, $t^{-1} S \in \mathscr{P}_{I}(S)$. Furthermore, $t\left(t^{-1} S\right) \neq 0$ since $\psi_{I}\left(T_{S}\right)$ is 0 -restricted and the claim follows. Thus, since $\Sigma(S) \in \mathcal{P}_{I}(S)$, we see from Lemma 4.5 and Theorem 3.7 (a) that $\Sigma(S) T^{1} \in \mathcal{P}_{I}(T)$. So, $\Sigma(T) \subseteq \Sigma(S) T^{1}$ by Lemma 7.2 and equality follows. Furthermore, since $\Sigma(T)=\Sigma(S) T^{1} \in \mathcal{P}_{I}(T)$ we see from Theorem 7.18 that $T$ is primitive dependent.

Corollary 7.23. Let $S$ be a primitive dependent $\mathcal{P}_{I}$-torsion free semigroup. Then $Q_{I}(S)$ is primitive dependent.

Proof. Since $Q_{I}(\Sigma(S)) \approx Q_{I}(S)$ by Lemma 7.19, and $\Sigma(S)$ is primitive dependent, we may assume without loss of generality that $S=\Sigma(S)$. Hence $S$ is embedded in $Q=Q_{I}(S)$ and $Q$ is a $\mathcal{P}_{I}$-torsion free $\mathcal{P}_{I}$-quotient semigroup over $S$. Furthermore, since $Q=\operatorname{Hom}_{S}(S, S)$ we have that $f s=f(s)$ for each $f \in Q, s \in S$. Therefore, $S$ is a left ideal of $Q$. Furthermore, by Theorem 4.2 of [8], $Q$ is regular. Hence the result follows from Theorem 7.22.

Our final theorem gives a complete characterization of all $\mathcal{P}_{I}$-torsion free primitive dependent semigroups. 
Theorem 7.24. Let $\Omega$ be an index set and for eacb $\alpha \in \Omega$, let $\mathcal{H}_{\alpha}^{0}=$

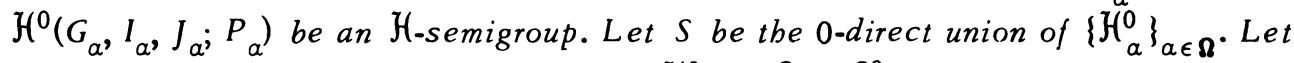
$T_{a}$ be any regular semigroup extension of $\mathcal{H}_{a}^{0}$ in $\mathcal{C}_{a}=\mathcal{C}_{a}^{0}\left(G_{a}, I_{\alpha}\right), a \in \Omega$, and let $T$ be any regular subdirect product of the $\left\{T_{a}\right\}_{a \in \Omega}$ over $S$. Then $T$ is a primitive dependent $\mathfrak{P}_{I^{-t o r s i o n}}$ free semigroup. Conversely, every primitive dependent $\mathscr{P}_{I^{-t o r-}}$ sion free semigroup is isomorphic to a semigroup obtained in this manner.

Proof. Since $\mathcal{C}_{a} \approx Q_{I}\left(\mathcal{H}_{\alpha}^{0}\right)$ over $\mathcal{H}_{\alpha}^{0}$ for each $\alpha \in \Omega$, we have that $\mathcal{C}_{a}$ is a $\mathscr{P}_{I}\left(\mathcal{H}_{\alpha}^{0}\right)$-quotient semigroup over $\mathcal{H}_{\alpha}^{0}$ which is $\mathcal{P}_{I}\left(\mathcal{H}_{\alpha}^{0}\right)$-torsion free. Thus $T_{\alpha}$ is a $\mathcal{P}_{I}\left(\mathcal{H}_{\alpha}^{0}\right)$-torsion free $\mathcal{P}_{I}\left(\mathcal{H}_{\alpha}^{0}\right)$-quotient semigroup for each $\alpha \in \Omega$. Hence by Theorem 6.7, $T$ is a $\mathcal{P}_{I}(S)$-torsion free $\mathcal{P}_{I}(S)$-quotient semigroup over $S$ and by Lemma 4.12 we see that $S \in \mathcal{P}_{I}\left(T_{S}\right)$. Thus, $T$ is also $\mathcal{P}_{I}(T)$-torsion free by Lemma 4.5 and Theorem 4.8. Since $\mathcal{H}_{\alpha}^{0}$ is a left ideal of $\mathcal{C}_{\alpha}$ for each $\alpha \in \Omega$, we have $\mathcal{H}_{\alpha}^{0}$ is a left ideal of $T_{\alpha}$. Let $t \in T$ and $s \in \mathcal{H}_{\alpha}^{0} \subseteq S$. Then

$$
t s(\beta)= \begin{cases}0 & \text { if } \beta \neq \alpha, \\ t(\alpha)_{s} \in \mathcal{H}_{\alpha}^{0} & \text { if } \beta=\alpha .\end{cases}
$$

Hence $t \hat{s}=\widehat{t}(\alpha) s$ and it follows that $S \approx \hat{S}$ is a left ideal of $T$. Therefore, the result follows by Theorem 7.22 .

Conversely, let $T$ be a $\mathcal{P}_{I}$-torsion free primitive dependent semigroup and let $S=\Sigma(T)$. Since $S \in \mathcal{P}_{I}(T)$ by Theorem $7.18(\mathrm{c}), S$ is $\mathscr{P}_{I}(S)$-torsion free. Hence by Theorem 7.16 we may assume that $S$ is a 0 -direct union of $\mathcal{H}$-semigroups $\mathcal{H}_{a}^{0}=$ $\mathcal{H}_{a}^{0}\left(G_{\alpha}, I_{\alpha}, J_{\alpha} ; P_{\alpha}\right), \alpha \in \Omega$. By Theorem 7.18(b) and Proposition 3.6,T is a $\mathcal{P}_{I}(S)$ quotient semigroup over $S$ which is $\mathcal{P}_{I}(S)$-torsion free. Thus, by Theorem 6.7 , there exist semigroups $T_{\alpha}, \alpha \in \Omega$, such that each $T_{\alpha}$ is a $\mathcal{P}_{I}\left(\mathcal{H}_{\alpha}^{0}\right)$-torsion free $\mathcal{P}_{I}\left(\mathcal{H}_{\alpha}^{0}\right)$-quotient semigroup over $\mathcal{H}_{\alpha}^{0}$ and $T$ is a subdirect product of the $\left\{T_{\alpha}\right\}_{\alpha \in \boldsymbol{\Omega}}$ over $S$. Since $\mathcal{C}_{\alpha}=\mathcal{C}^{0}\left(G_{\alpha}, I_{\alpha}\right)$ is isomorphic over $\mathcal{H}_{\alpha}^{0}$ to $Q_{I}\left(\mathcal{H}_{\alpha}^{0}\right)$, we may choose the $T_{\alpha}$ such that $\mathcal{H}_{\alpha}^{0} \subseteq T_{\alpha} \subseteq \mathcal{C}^{0}\left(G_{\alpha}, I_{\alpha}\right)$ by Corollary 5.8. Furthermore, each $T_{\alpha}$ is regular since it is the homomorphic image under the projection map of the regular semigroup $T$. Therefore, the theorem follows.

This paper constitutes part of the author's doctoral dissertation written under the direction of Professor D. R. LaTorre. The author wishes to thank Professor LaTorre and Professor John K. Luedeman for the ir valuable advice and encouragement.

Research for this work was supported by a National Science Foundation Traineeship and by a Faculty Fellowship from the Virginia Military Institute.

\section{REFERENCES}

1. K. Asano, Über die Quotientenbildung von Schiefringen, J. Math. Soc. Japan 1 (1949), 73-78. MR 11, 154. 
2. Gordon L. Bailes, Right inverse semigroups, Thesis, Clemson University, Clemson, S.C., 1972.

3. P. Berthiaume, The injective envelope of S-sets, Canad. Math. Bull. 10 (1967), 261-273. MR $35 \# 4321$.

4. A. H. Clifford and G. B. Preston, The algebraic theory of semigroups, 2nd ed., Vol. I, Math. Surveys, no. 17, Amer. Math. Soc., Providence, R.I., 1961. MR 24 \#A2627.

5. - The algebraic theory of semigroups. Vol. II, Math. Surveys, no. 17, Amer.

Math. Soc., Providence, R.I., 1967. MR 36 \#1558.

6. E. H. Feller and R. L. Gantos, Indecomposable and injective S-systems with zero, Math. Nachr. 41 (1969), 37-48. MR 40 \#7374

7. T. E. Hall, On regular semigroups whose idempotents form a subsemigroup, Bull. Austral. Math. Soc. 1 (1969), 195-208. MR $40 \# 2772$.

8. C. V. Hinkle, Jr., The extended centralizer of an S-set (submitted).

9. R. E. Johnson, The extended centralizer of a ring over a module, Proc. Amer. Math. Soc. 2 (1951), 891-895. MR 13, 618.

10. F. R. McMorris, On quotient semigroups (submitted).

11. - Vital injective S-systems, Math. Nachr. 47 (1970), 121-125. MR 44 \#342.

12. - The singular congruence and the maximal quotient semigroup, Canad.

Math. Bull. (to appear).

13. Mario Petrich, Topics in semigroups, Pennsylvania State University, 1967.

14. David A. Smith, On semigroups, semirings, and rings of quotients, J. Sci.

Hiroshima Univ. Ser. A-I Math. 30 (1966), 123-130. MR 34 \#692.

15. Yuzo Utumi, On quotient rings, Osaka Math. J. 8 (1956), 1-18. MR 18, 7.

DEPARTMENT OF MATHEMATICS, CLEMSON UNIVERSITY, CLEMSON, SOUTH CAROLINA 29631

DEPARTMENT OF MATHEMATICS, VIRGINIA MILITARY INSTITUTE, LEXINGTON, VIRGINIA 24450 (Current address) 